This item was submitted to Loughborough's Research Repository by the author.

Items in Figshare are protected by copyright, with all rights reserved, unless otherwise indicated.

\title{
WABI: Facilitating synchrony between inhabitants of adaptive architecture
}

PLEASE CITE THE PUBLISHED VERSION

https://www.springer.com/gb/book/9783319708744

PUBLISHER

Springer

VERSION

AM (Accepted Manuscript)

PUBLISHER STATEMENT

This is a pre-copyedited version of a contribution published in Schnadelbach, H. and Kirk, D. (eds.), People, Personal Data and the Built Environment, published by Springer. The definitive authenticated version is available online via http://doi.org/10.1007/978-3-319-70875-1

\section{LICENCE}

CC BY-NC-ND 4.0

\section{REPOSITORY RECORD}

Jager, Nils, Holger Schnadelbach, Jonathan Hale, David Kirk, and Kevin Glover. 2019. "WABI: Facilitating Synchrony Between Inhabitants of Adaptive Architecture". Loughborough University. https://hdl.handle.net/2134/35670. 


\title{
WABI: Facilitating Synchrony between Inhabitants of Adaptive Architecture
}

\author{
Nils Jäger ${ }^{1}$, Holger Schnädelbach², Jonathan Hale ${ }^{3}$, David Kirk ${ }^{4}$, Kevin \\ Glover $^{2}$ \\ ${ }^{1}$ School of Architecture, Building and Civil Engineering, Loughborough University, \\ Leicestershire, LE11 3TU, UK \\ ${ }^{2}$ Mixed Reality Laboratory, School of Computer Science, University of Nottingham, Jubilee \\ Campus, Wollaton Road, Nottingham, NG8 1BB, UK \\ ${ }^{3}$ Department of Architecture and Built Environment, School of Engineering, University of \\ Nottingham, Paton House, University Park, NG7 2RD, UK \\ ${ }^{4}$ Department of Computer and Information Science, CIS Building, Northumbria University, \\ Newcastle upon Tyne, NE1 8ST, UK
}

\section{Abstract}

We spend most of our lives in buildings where we interact with people that occupy the same space. A common and intuitive form of interaction with others is to synchronise our own behaviour with theirs and such interpersonal synchrony can have various benefits for our wellbeing. We present research that investigates how a new prototype of digitally-driven adaptive architecture called WABI facilitates behavioural synchrony between its inhabitants. We designed three interaction modes, which each feature a unique mapping and processing of physiological data emanating from inhabitants. Qualitative feedback from a first exploratory study indicates that the different interaction modes affect how inhabitants interact and synchronise their behaviours. We discuss how adaptive architecture might contribute to wellbeing, therapy, and sports by facilitating synchrony.

Keywords: Adaptive Architecture, synchrony, embodied interaction, data mapping, empirical study 


\section{Introduction}

When Mark Weiser (1991) described his vision of computers disappearing into the background surrounding us, he painted a picture of refreshing interactions with computers similar to "taking a walk in the woods." Focusing on an individual (see scenario describing a day in the life of Sal), it remained less clear in this picture how much and what kind of data these embedded, ubiquitous computers would (need to) collect about us. Largely unaddressed was how multiple co-present people would interact with each other and the computerised environment at the same time beyond singular events, such as sharing biographical information with each other.

Bringing the challenge of multiple people interacting with each other into focus, we present an adaptive architectural prototype that uses physiological data emanating from its inhabitants to facilitate synchronous behaviour between them.

\subsection{Synchrony}

Interpersonal synchrony, 'the coordination of movement between individuals in both timing and form...' (Kimura and Daibo 2006), is a form of interaction with which we are intimately familiar. According to Chartrand and van Baaren (2009), coordinating our own behaviour with that of others can occur as both conscious imitation of others as well as nonconscious alignment of behaviours. The authors argue that such coordination (or synchrony) is ubiquitous in our lives and plays an important role in how we communicate with each other.

Already as unborn babies, we occasionally synchronise our heart rates with that of our mothers (Van Leeuwen, Geue, and Lange 2003). Indeed we learn about the world by synchronising our behaviours with that of our parents, as for example, Feldman (2007) explains. Among others, synchrony with their mother increases the social-emotional development (Feldman and Eidelman 2004) and leads to improved self-regulation (Feldman, Greenbaum, and Yirmiya 1999) in children.

The benefits of synchrony continue into adulthood. For example, interpersonal synchrony can positively affect social perception (Miles, Nind, and Macrae 2009) and improve one's memory (Miles et al. 2010). It can increase one's self-awareness and other-awareness (Asendorpf, Warkentin, and Baudonnière 1996) and can also improve one's work performance while lowering state anxiety (Sanchez-Burks, Bartel, and Blount 2009) to name but a few positive outcomes of synchrony.

\subsection{Synchrony in Social Situations}

Specific social situations are relevant for the discussion of synchrony in the context of architecture. In a variety of areas and spaces, studies have shown the positive effects of synchronised behaviour. For example, in sports, and specifically rowing, Cohen et al (2010) correlated behavioural synchrony with an increased pain threshold. Ramseyer and Tschacher (2011) showed that higher levels of nonverbal synchrony between therapist and client reduced client symptoms in a psychotherapy 
setting. Sanchez, Bartel, and Blount (2009) provide evidence that interpersonal synchrony affects work performance depending on cultural group membership in an office environment. These examples illustrate that synchrony can occur in specific social situations and physical spaces, namely in sport (e.g., in a gym), in therapy (e.g., treatment rooms), and in the workplace (office spaces).

\subsection{Influencing Occupant Behaviour}

Numerous studies have shown that the physical environment affects the behaviour of people. While the initial understanding was that of a deterministic relationship between design and behaviour (Marmot 2005), it now seems more likely that this relationship is rather probabilistic (cf. Bell et al. 1996). Evidence gathered in retail suggests that the manipulation of environmental factors, such as atmospherics (Turley and Milliman 2000), music (Yalch and Spangenberg 2000), scent (Spangenberg, Grohmann, and Sprott 2005), and spatial arrangement (Smith and Burns 1996) influences how we evaluate products and our likelihood to buy them. Thus, architectural spaces and elements within them have been shown to have the potential of affecting occupant behaviour.

\section{Adaptive Architecture as Interaction Partner}

In an even more deliberate and immediate fashion, adaptive architecture can interact directly with its inhabitants. Adaptive Architecture is defined as buildings that have been specifically designed to adapt to their environments and inhabitants (Schnädelbach 2010). Technology is now enabling architecture to become increasingly behaviour-responsive, which can be traced through several publications, such as Bier and Knight's (2010) Footprint issue on digitally-driven architecture, Bullivant's (2005b; 2006) work on responsive and interactive environments, Fox and Kemp's (2009) book Interactive Architecture, Kolarevic's (2015) book titled Building Dynamics, Architectural Robotics (Green 2016), and most recently the book Architecture and Interaction (Dalton et al. 2016). These provide numerous cases in which adaptive architecture directly or indirectly affects the behaviour of its inhabitants.

\subsection{Interactions between Architecture and Inhabitants}

The growing number of adaptive designs and research projects makes apparent that architecture is increasingly developing into an active participant in human activities. However, the built examples of interactive architecture (described in the publications above and individually published) tend to feature two main modes of response to inhabitant behaviour. With very few exceptions, such as Lungs the Breather (Guerra, Todoroff, and Sebti, n.d.) or ADA (Eng et al. 2003), current adaptive environments respond to (1) a single occupant or (2) they aggregate behaviour of multiple occupants into one response for all, both of which we discuss below. 


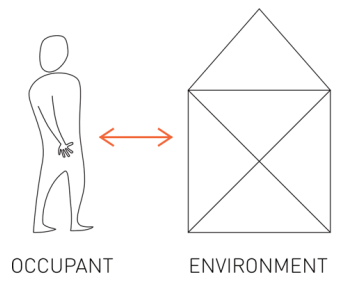

(A)

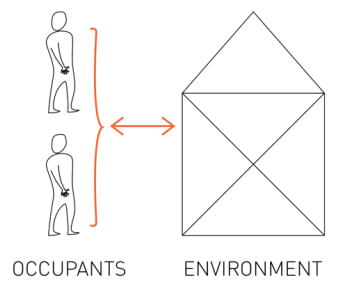

(B)

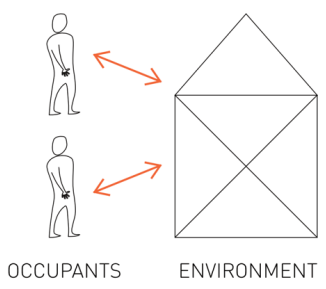

(C)

Figure 1: Illustration of response types of Adaptive Architecture. (a) single inhabitant - single response; (b) multi-inhabitant - single response; (c) multi-inhabitant - multiple responses. Orange arrows indicate direction of interaction.

Particularly well understood are interactions between adaptive architecture and a single inhabitant (Figure 1, a). Indeed, most real-time adaptive architectural spaces, especially those employing kinetic elements, respond to one person at a time, such as the original ExoBuilding (Schnädelbach, Glover, and Irune 2010), Breathe (Jacobs and Findley 2015), Sonic Cradle (Vidyarthi, Riecke, and Gromala 2012), Bug (K. Najjar and Najjar 2016) or Reciprocal Space (Glynn 2005) to name a few. In all these examples, a single inhabitant interacts with the physical structure surrounding her or him - though Sonic Cradle offers an interactive soundscape, not a physical actuation.

\subsubsection{Synchrony between Inhabitant and Adaptive Environment}

An experimental study of the kinetic biofeedback environment ExoBuilding (Schnädelbach et al. 2012) showed the embodied relationship between individual inhabitant and adaptive environment: when the structure synchronised its movement with the breathing of its inhabitant by moving up (inhalation) and moving down (exhalation), inhabitants tended to breathe slower, deeper, and more regularly compared to seeing the environment move automatically or not move at all. Another study by Jäger et al. (2017) showed that the embodied nature of the interaction between inhabitant and ExoBuilding could be used to reverse the control relationship, leading inhabitants to synchronise with ExoBuilding and slow their respiratory rates.

\subsubsection{Adaptive Architecture Interacting with Multiple Inhabitants}

The most common mode of multi-inhabitant interactions is that of aggregating data from numerous inhabitants to which the environment generates a single response as shown in Figure 1, b. Examples of this form of interaction are Sky Ear (Bullivant 2005a), D-Tower (Bullivant 2005c), and Open Columns (Khan 2010). The latter, for example, respond to differences in carbon dioxide $\left(\mathrm{CO}_{2}\right)$ levels in an interior space. When $\mathrm{CO}_{2}$, a product of exhalation, reaches a threshold, a column slowly drops from the ceiling and disperses the people gathered underneath. 
Unlike the above projects which respond to aggregate user data , only $A D A$ (Eng et al. 2003) so far represents multi-inhabitant/multi-response environments. $A D A$ could, for example, trace the walking path of multiple individuals, while also responding with audio messages to different individuals. Thus, $A D A$ reveals a new complexity in interactions with its inhabitants, which are the potential overlaps of responses. For example, audio and visual responses might clash or two audio responses overlap such that the meaning of either response becomes compromised or even lost.

This form of Adaptive Architecture which responds to each of its inhabitants individually and the interaction system that emerges, as shown in Figure 1, c, is currently underexplored. No study exists on how such interaction systems affect interpersonal synchrony.

Of particular interest are the questions of (1) how such adaptive environments affect interpersonal synchrony, and (2) how different data mappings affect the interaction system of adaptive architecture and its inhabitants. Understanding the principles and effects of this interaction system and the interplay of data mappings and interpersonal synchrony will enable designers to create meaningful interactions between inhabitants and adaptive environments to the benefit of inhabitants. Application areas will likely reside in healthcare and wellbeing, including physical and behavioural therapy, relaxation activities, and targeting the other positive individual and inter-personal effects emerging from interpersonal synchrony discussed above.

\section{WABI: Designed to Facilitate Interpersonal Syn- chrony}

To investigate the facilitation of synchrony between inhabitants - and more generally the architectural responses to and interactions with multiple individual inhabitants (Figure 1, c) - , we designed WABI (Figures 2 and 3) as an instance of embodied adaptive architecture and based it on the principles of Schnädelbach et al.'s (2012) and Jäger et al.'s (2017) experimental work. WABI is a digitally-driven adaptive environment for two inhabitants that uses sensors and actuators to kinetically respond to the physiological behaviour (heart rate and breathing) of both inhabitants in real time and individually via a biofeedback mechanism (see Schnädelbach 2011 for a detailed explanation of the biofeedback loop), which establishes a coupling between each inhabitant and the environment, as well as between inhabitants.

Specifically, the physiological mechanism driving WABI is called heart rate variability, identical to Jäger et al.'s (Jäger et al. 2017) study. We chose this mechanism specifically as it is a well-established biofeedback technique to improve relaxation through breathing techniques, for example used in consumer devices, such as StressEraser (Muench 2008). 
Heart rate variability is the phenomenon of a person's heart rate naturally fluctuating (Berntson et al. 1997). Heart rate variability can be affected through breathing. The emerging process is called respiratory sinus arrhythmia, which means that heart rate responds to breathing patterns (Hirsch and Bishop 1981; Yasuma and Hayano 2004): inhalation causes the heart to beat faster, an increase of heart rate, while exhalation causes the heart to beat slower, a decrease of heart rate. This effect is most pronounced at slow respiratory rates. However, the optimal rate varies by individual and can be slower for some than for others. To achieve pronounced heart rate variability, participants were told to breathe at a comfortably slow rate and pay particular attention to exhaling, while counting slowly to four.

WABI responds to heart rate by moving up when heart rate increases and moving down when heart rate decreases. Participants experience that inhaling causes the environment to rise, while exhaling causes the environment to fall.

\subsection{Physical Setup}

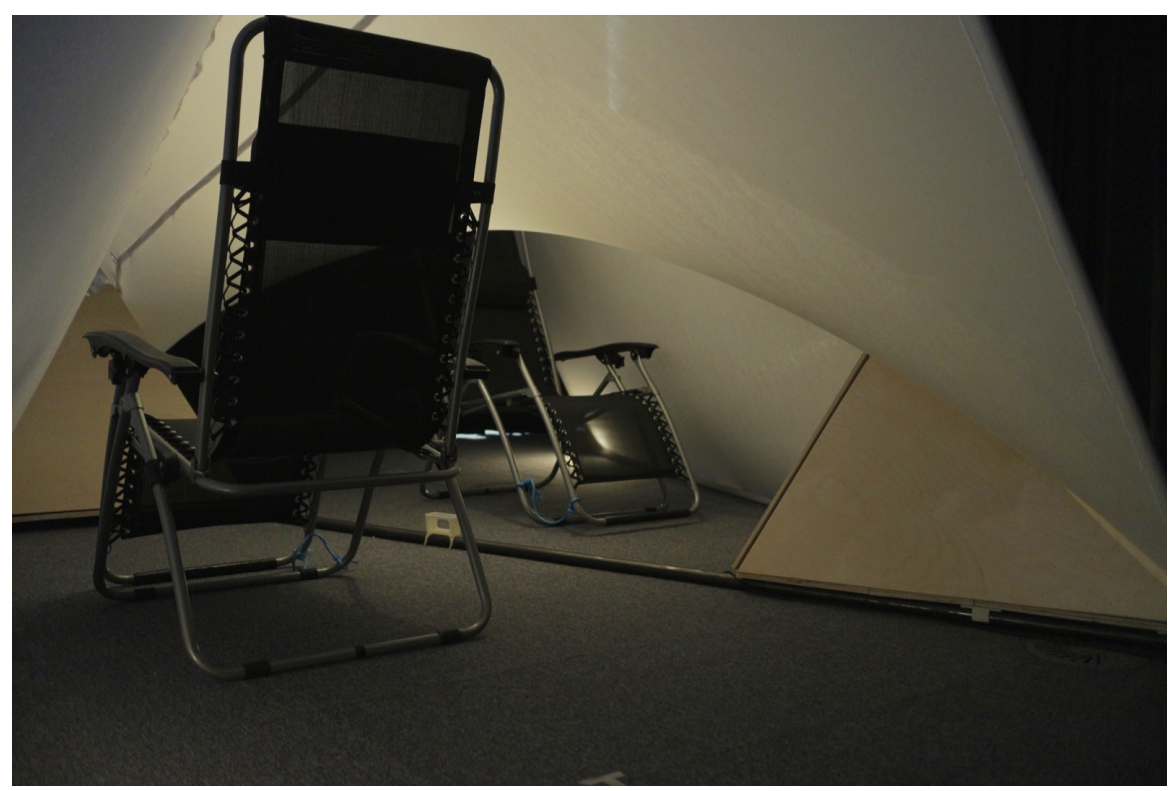

Figure 2: Interior view of WABI showing relationship between inhabitants. 


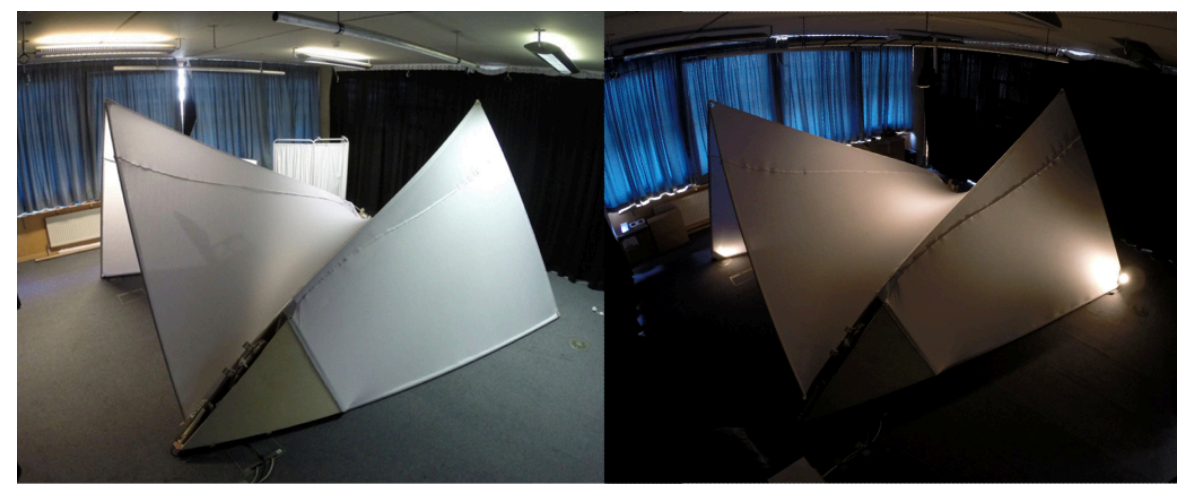

Figure 3: Exterior view of WABI (left: overview; right: soft lighting for experiment).

To measure breathing and heart rate reliably, inhabitants need to sit throughout their interaction (Figure 2). They sit facing each other in reclining chairs, each positioned inside one of two tetrahedral segments that are 180-degree tessellated. The sharp end of each building section contains the movement mechanism, opposite which inhabitants can enter the softly lit steel frame structure (Figure 3; see also supplemental material for a detailed description).

\subsection{Interactions with WABI and the Co-inhabitant}

The design of WABI is based on principles to facilitate synchrony both spatially and technologically. To establish interpersonal synchrony humans rely on perceiving behavioural cues of their interaction partner (Chartrand and van Baaren 2009). Thus, we designed WABI to enable perception of both audible and visual cues (Fouriezos et al. 2007) from the environment as well as the interaction partners. Visual cues are created by having inhabitants face each other (see Fig. 2). Movements of the partner are easily visible. Audible cues are a result of the proximity within and the intimacy of the shared space, enabling inhabitants to communicate verbally or simply hear their partner's breathing. Additionally, both inhabitants sit near either of the motors. This exposes them to the sounds of the operating servo motors, providing another cue of either their own or their partner's behaviour. In a previous study of a different prototype, several efforts had been made to eliminate sound as a variable using insulation around servo motors as well as noise-attenuating headphones. Participants reported that they could still hear the motors and on average found the sound helpful as additional feedback source. Finally, inhabitant behaviour is reflected through the upward and downward motion of WABI's flexing 'spines' of about $15 \mathrm{~cm}$.

Based on the predominant data processing approaches in adaptive architecture described above (single user/single response and multi-user/aggregate-response), we designed three interaction modes with implicit goals to investigate how they would affect synchrony. 


\subsubsection{Interaction Modes and Expectations}

The three interaction modes (Fig. 4) used for this study reflect the usual data processing methods of (a) individual feedback to a single user, (b) aggregate feedback to multiple users. A final mode is, to the best of our knowledge unique in the field of responsive architecture: (c) individual feedback crisscrossed between users.

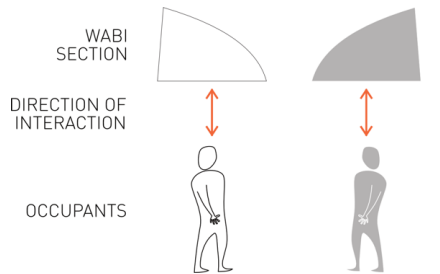

(A)

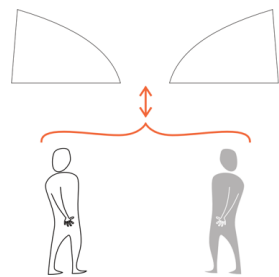

(B)

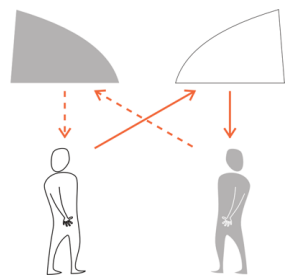

(c)

Figure 4: Graphic representation of WABI's interaction modes and data mappings; (a) Own Feedback: inhabitant data drives section surrounding inhabitant, (b) Aggregate Feedback: each inhabitant contributes 50 percent to system, environment creates single response, (c) Crisscross Feedback: inhabitant data drives partner's section. Top row: WABI sections; orange arrows: direction of interaction; bottom row: occupants.

\section{Own Feedback Interaction}

Each inhabitant's heart rate drives the building section directly surrounding them (Figure 4, a). Data of inhabitant A is mapped to building section A, inhabitant B to building section B. Thus, both inhabitants experience feedback of their own behaviour in proximity to themselves.

\section{Crisscross Feedback Interaction}

The signals of both inhabitants have been swapped (Figure 4, b): inhabitant A is mapped to building section $\mathrm{B}$, and inhabitant $\mathrm{B}$ to building section $\mathrm{A}$. The signals cross over between adjacent parts of the environment.

\section{Aggregate Input Interaction}

Inhabitants A and B collectively drive building sections A and B, each having 50 per cent input into the interaction (Figure 4, c). When they synchronize their physiological behaviours, WABI moves both its sections simultaneously. 180 degrees inverted synchrony means that the two physiological signals cancel each other out and WABI does not move at all. 


\subsubsection{Expectations}

Each of the interaction modes had implicit goals that related both to the specific data processing as well as the physical setup. Own Feedback replicated interactions seen, for example, in ExoBuilding (Schnädelbach, Glover, and Irune 2010) and Sonic Cradle (Vidyarthi, Riecke, and Gromala 2012). Since there was no implicit interaction between inhabitants, our expectation was that this would lead inhabitants to focus on their own behaviour. Crisscross Feedback implied that inhabitants were exposed to (or immersed in) their partner's data. Thus, we expected them to either ignore their partner's behaviour or align themselves with it. Aggregate Feedback, for example used in Open Columns (Khan 2010) or Sky Ear (Bullivant 2005a), implied a togetherness or combined effort. Accordingly, we expected participants to overtly coordinate their behaviour to create a meaningful interaction.

In alignment with our expectations of inhabitant behaviour, we anticipated measurements of synchrony (self-report and physiological data) to be highest in Aggregate Feedback mode and lowest in Own Feedback mode.

\section{Study}

\subsection{Methods}

We used WABI as the experimental environment, employing the three described interaction modes. The aims of this study were to explore (1) WABI as a prototypical shared biofeedback environment and (2) the interactions between inhabitants as mediated by the environment. More specifically, we wanted to assess if any of the three different interaction modes described above particularly encouraged physiological and/or behavioural synchronization between participants.

\subsubsection{Participants}

We recruited 16 participants (5 Female, 11 Male) between 19 and 36 years old with a mean age of $25.06(S D=4.057$; Mode $=24)$ through department-wide email distribution. They were students (undergraduate and postgraduate), and an officer (term reported by participant). Participants' ethnic backgrounds were Asian (5), Caucasian (7), African (1), and Other (3). Conditions for participation were to be generally healthy (no heart conditions or respiratory problems), not to be claustrophobic, and not to have experienced any of our other adaptive environments. Participants were compensated for their time with a retail gift certificate. The 16 participants formed 8 pairs out of which two consisted of strangers, four pairs indicated that they were friends, and two pairs were romantic partners.

Out of the eight recruited pairs, two had to be removed from analysis due to technical problems relating to the movement mechanism of WABI. This left six pairs (12 participants) for the analysis. We deemed 6 pairs (12 participants) to be a sufficiently large sample (for example discussed by Crabtree, Tolmie, and Rouncefield, 2013) to gain an adequate range of behaviours and user experiences as basis 
for further work. Qualitative and quantitative studies of similar prototypes used comparable numbers of participants, such as Sonic Cradle (15 participants), ExoBuilding (12 participants), and ExoPranayama (4 phases, each between 2 and 12 participants).

\subsubsection{Procedure}

The study, which was approved by an internal ethics review board, consisted of three discrete experiences representing the three different interaction modes with WABI. These modes - own, crisscrossed, and aggregate feedback - were chosen as being representative of a variety of data manipulations possible in collocated interactions with adaptive architecture as discussed above.

The sequence in which participants experienced the modes was kept the same for all pairs. In pilot trials, participants told us that the easiest interaction mode to comprehend was the Own Feedback mode, followed by the Crisscross Feedback mode. The most challenging trial, they said, was the Aggregate Feedback mode. Because of the exploratory nature of this study, we wanted participants to slowly build experience and expertise before engaging with the most challenging interaction mode. Thus, we made a conscious decision not to counterbalance the sequence to avoid unnecessary frustration and potential premature disengagement of participants.

Participants were run in pairs who experienced each trial as an eight-minute long period of sitting in a reclined chair inside WABI.

Figure 5 shows a graphical representation of the session. The vertical axis on the left represents progress through the session, while the horizontal boxes labelled (1), (2), and (3) represent the experience of the different interaction modes, each 8minutes (net) long. 


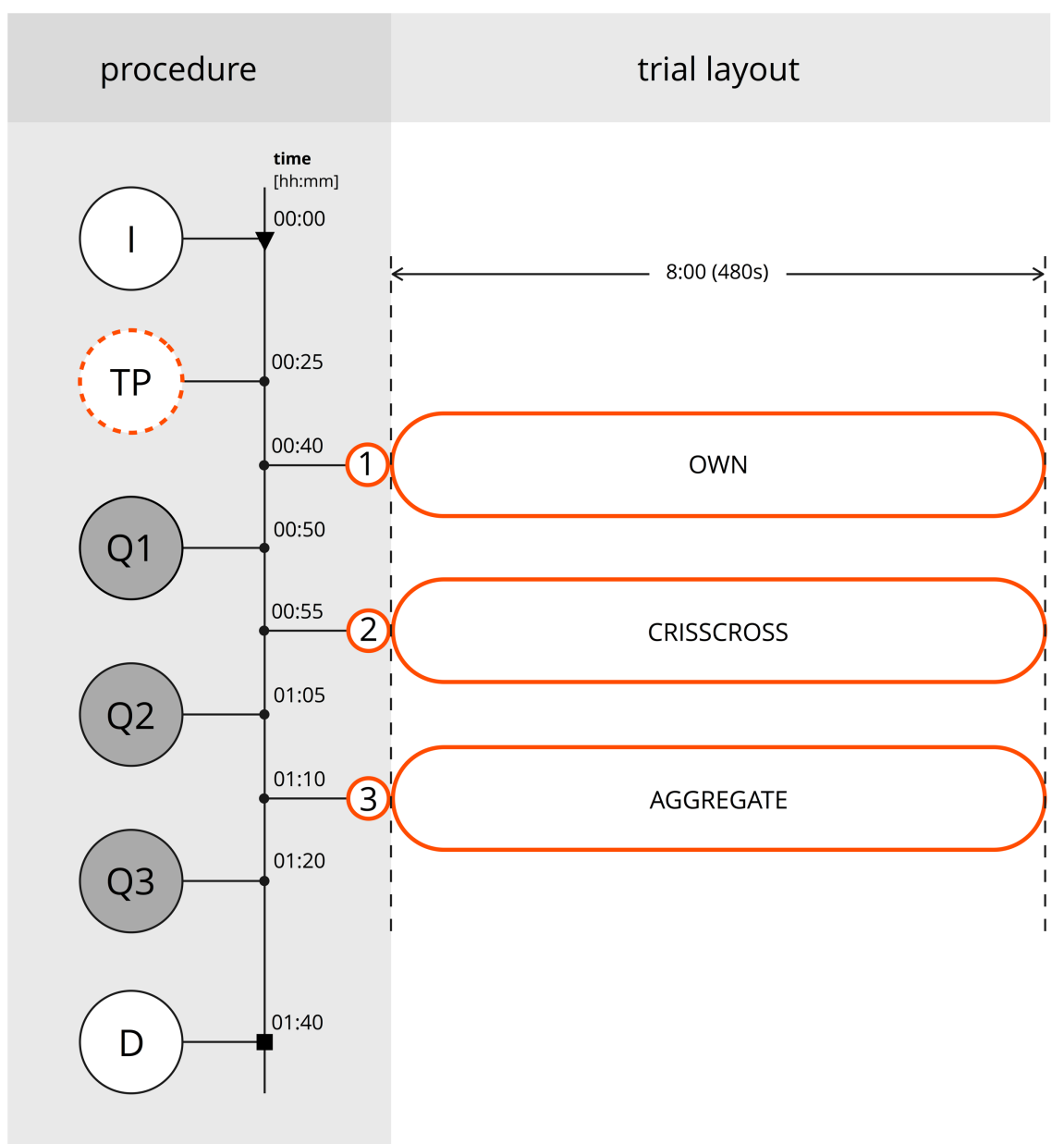

Figure 5: Procedure (time indicated in hh:mm). I: introduction; TP: Test Phase; 1: Trial 1 (own); 2: Trial 2 (crisscross); 3: Trial 3 (aggregate); Q1: Synchrony Questionnaire and Post-trial Interview 1; Q2: Synchrony Questionnaire and Post-trial Interview; Q3: Synchrony Questionnaire and Posttrial Interview; D: Final Interview and Debrief

Before the first trial, participants were fitted with a medical grade bio-sensing unit (see below). Then, they individually familiarized themselves with the environment and the physiological control mechanism for about 3 minutes for which we used the Own Feedback Mode.

Following this familiarisation, the experimenter explained the interaction mechanism and the three interaction modes. After each of the three trials $(\mathrm{Q} 1, \mathrm{Q} 2$, and Q3), we asked participants to answer a short questionnaire to assess their self-perceived synchrony. We do not report on this questionnaire due to the small sample size and to avoid misunderstandings of WABI's effects on inhabitants. Following the questionnaires, a mini interview consisting of three questions (see Appendix) 
was conducted while participants remained seated in WABI. Before each trial, participants were reminded of the interaction mechanics of the upcoming trial. After all three trials had completed, we conducted a final, longer interview and debriefed participants.

\subsection{Measurements}

The experimenter fitted each participant with a respiration belt (RSP), electrocardiogram (ECG) electrodes and galvanic skin response (GSR) electrodes, which collected data at a rate of 32 samples per second. These are part of the NeXus-10 biosensing unit by MindMedia (2015). Due to unreliable data from the GSR electrodes, we do not include this data here. Participants were also asked to complete a micro questionnaire about synchrony after each trial. We also conducted semi-structured, audio- recorded mini-interviews after each trial. In Q3 (Figure 5), we conducted a longer semi-structured, audio- recorded interview to investigate the relationship (1) between inhabitants and WABI and (2) between inhabitants.

\subsection{Results}

The report of results is structured by interaction mode, beginning with Own Feedback Interaction. The results consist of interview data and reports of qualitative physiological data. Observations from video recordings of the trials support both the physiological data where appropriate.

\subsubsection{Own Feedback Interaction}

During the interviews, only two (2/12) participants told us that they had preferred this first trial to the other trials. Both had very similar - arguably more individualistic - reasons, with one of them (P04-2) perceiving 'more freedom' in this trial, as 'it did not give the pressure to deal with' another person. The other participant ( $\mathrm{P05}-$ 1) enjoyed moving his own environment compared to the other interaction modes. Two other participants indicated that they had liked receiving their own feedback as well, though both indicated Crisscross Feedback Interaction (Trial 2) as their preference. However, they explained that their liking of trial 1 applied to being alone (P08-2) and allowed them better to 'play with the experience' (P09-2).

In terms of interactions between the partners, participants tended to talk about establishing eye contact with their partner, trying to avoid it, or their perception of

1 The questions included (1) Please tell me about your experience in this trial. (2) What did you mainly focus on? (3) Did you interact with each other?

2 The questions of the concluding interview were probing (1) participants' first impressions of WABI, (2) their relationship to WABI, (3) how they established smooth and gentle motion of WABI, (4) if they were able to synchronize, (5) which interaction mode they preferred, (6) what their expectations were , (7) if they communicated with each other in any form, (8) what they think about a building that reacts to their body, (9) what they think about sharing physiological data with an everyday building, (10) how they feel about future buildings collecting data all the time, and (11) what they would change about WABI. 
Trial 1 as being an individual experience that 'precluded interaction' with their partner (P08-2). Both participants of pair P05 agreed on 'not having looked at each other' or not having interacted. Other pairs disagreed in their assessment of this trial as indicated by pair 03 for whom P03-1 explained to have 'looked over to [P03-2] many times'. P03-2, however, stated that the fabric between the two building sections was initially too low to see his partner and subsequently he 'did not try to look over again'. Similarly, pairs 07 and 09 both stated that one of the pair looked to their partner, which the other did not reciprocate or even actively 'tried to avoid eye contact and interaction' (P07-2) knowing that his partner would start to laugh. More explicitly, P07-2 described the experience as '[...] solo, where I was responsible for my own environment.'

Physiologically, no pair achieved sustained synchrony - three or more cycles in their respiratory behaviour despite being able to finely tune their own respiration patterns. For example, respiration traces of pair 07 (Figure 12) reveal that both occupants achieved consistently sustained regular breathing for most of the trial. Especially P2's breathing pattern is regular regarding both frequency-between five and six breaths per minute - and amplitude. Comparing all trials, P07-2 thought that this 'first one took a lot longer to sync.'

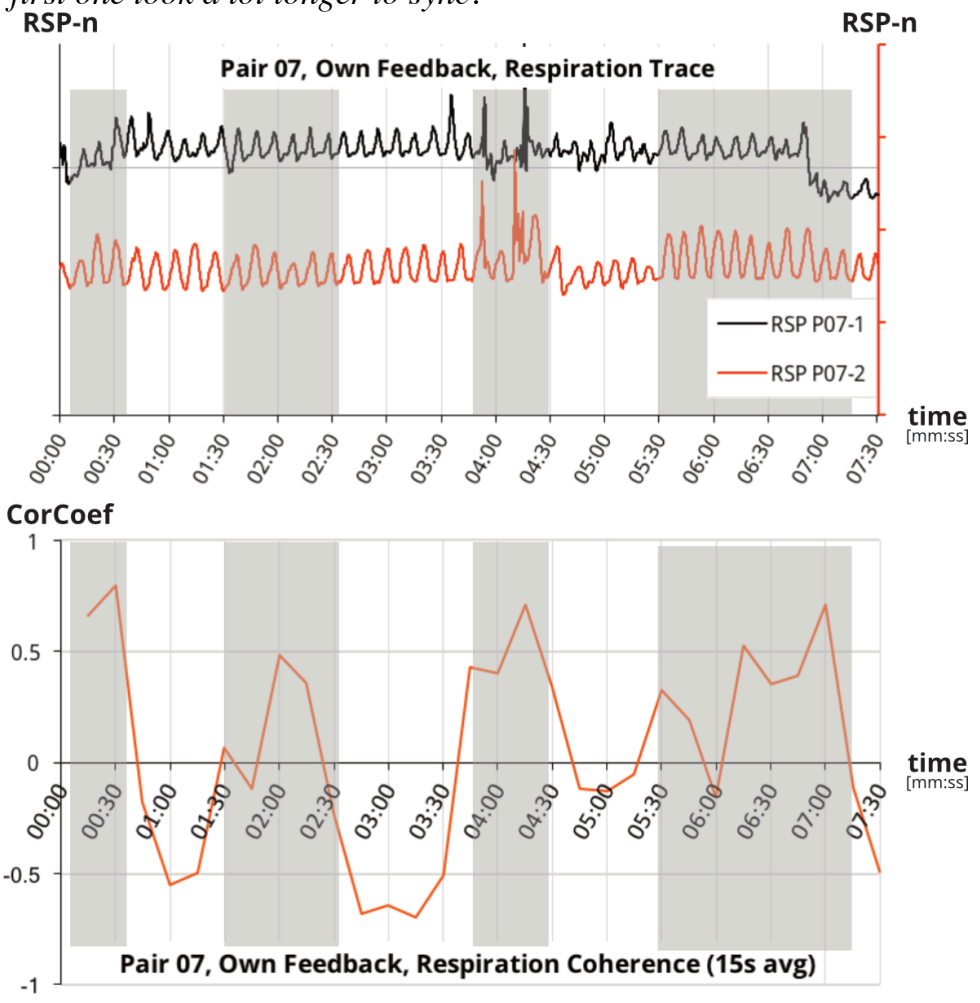


Figure 6: Respiration trace (upper graph, showing raw, normalised sensor data) and between-inhabitant Respiration Coherence (lower graph, showing Pearson Correlation Coefficient) of pair 07 in Own Feedback Interactions over trial duration. Both respiration traces (upper graph) show extensive periods of auto-coherence, with each participant breathing slowly and regularly. They correlate occasionally with each other (grey highlights). Only correlations (lower graph) above 0.5 are considered statistically significant.

In summary, Own Feedback Interaction was perceived as an individual experience, in which participants felt alone in their own section of WABI with very little or no interaction with their partners. Accordingly, most participants focused on their own respiration, while they were only peripherally aware of their partner's presence and explained not to have engaged in interactions with their partners. Thus, the location of the architectural response in immediate vicinity of the occupant appears to significantly reduce the sense of presence of another, collocated person.

\subsubsection{Crisscross Feedback Interaction}

Most participants (7/12) preferred crisscross feedback interactions because of the unexpectedness of the sensation of swapping feedback signals between the two sections of the environment (P03-1), the triggering of alertness (P05-2) and the increased awareness of the partner (P05-2, P07-2), the fun of controlling someone else's environment alongside the challenge to synchronize (P07-1), and the shared goal or focus and that synchronization with the partner occurred naturally (P09-2). Pair 08 enjoyed this the most as it allowed them to connect with their partner's section of WABI, and, thus, created a deeper connection with the environment (P081). P08-2 found the opportunity to have personal, playful goals, such as 'messing with Pl's environment' most interesting.

One participant (P03-2) even felt a 'sense of responsibility' for their partner's experience because of the specific mapping of the physiological signals. This link to another person's experience was also expressed by P07-1 who found it fun to be able to have control over someone else's environment and found it easy to synchronize breathing behaviours:

' $[\mathrm{In}]$ the 2nd [trial], it was pretty easy because you knew [the partner's section of WABI] was making that sound. This is the one you're visualising and that's [the partner's]. Then, you'd get more into your own environment, so the other person's breathing. [...]'

Crisscross Feedback Interactions stimulated interactions more than we expected. For example, many participants tried to synchronize with their partner due to its apparent ease. P07-1:

'It's like when you're singing in a choir. You want everyone to be singing the same thing at the same time, rather than at different paces at different times. Because then it gets confusing. When we were doing it together, it was so much easier to do it at the same time. It's quite interesting that we were able to influence each other's breathing.'

P07-2 supported this perception of synchronization in this trial, saying: 
'[...] With the 2nd one, you could see how they're breathing while inherently hearing the motor [...] going up and down for your [own], which meant you had a lot more information to work with. Then, you could [...] synchronise a lot easier.'

The increased interactions also led to pair 07 feeling more connected to each other. P07-1 explained that she thought about 'how my environment is your [environment] and how that compares to the one that I am in right now.' P07-2 explicitly made the point of a closer connection to his partner, explaining:

'The 2nd [trial] felt a lot more connected, where someone is in charge of your environment and you're in charge of theirs, which meant you, after visually seeing the environment change, you'd kind of connect and have a somewhat similar breathing rate.'

Others seemed to enjoy their experience more, playfully exploring various behaviours. For instance, P08 tried to play with each other's environments, with P081 explaining that she wanted to 'make his tent dance' and P08-2 saying he had tried to 'keep her tent down' because according to his knowledge P08-1 is 'a little claustrophobic.'

Another account of perceived synchrony was provided by pair 09 who described that they thought they had synchronised with each other during the second half of this trial. The following excerpts from the interview highlight the matter of synchronising between both partners, the point in time when this occurred, and how they related to $\mathrm{WABI}$ :

P09-1: I think in the second trial, we sort of synced at the end, in the second half of the trial.

Interviewer: Did you do anything special? Or did it naturally emerge?

P09-2: I was watching the environment.

P09-1: Yes, me too. Like paying attention to both environments.

P09-2: I was just following you.

P09-1: No, you didn't.

P09-2: But you said you were following me.

P09-1: Yes... Because we were controlling each other's environment. So, we sort of also respond to your own environment, [...].

P09-1 clearly identified the time when the synchronisation happened as being during the second half of the trial. The excerpt also illustrates how the crisscrossing of feedback enabled participants to relate to each other via WABI as it allowed them to watch the behaviour of the environment and, thus, the behaviour of their partner, while also controlling their breathing in relation to their partner.

As the physiological data of pair 09 (Figure 7, top graph) shows, at time 03:45, P09-1 (black graph) suddenly changed the breathing pattern to match that of P09-2 exactly. P09-1 had already established an auto-coherent breathing pattern during the first half of the trial. At 03:45, she changed her breathing pace dramatically from about 10-11 cycles per minute to a respiratory pace of about six cycles per minute, an effective reduction of nearly 50 per cent. The RSP Correlation (see Figure 7, bottom graph) between the two participants also shows how, from the mid-point of the trial, both signals become highly correlated, consistently above correlations of 0.5 -statistically values above 0.5 are considered highly correlated (cf. J. Cohen 
1992). On two occasions, they even reach nearly perfect correlation, with values of 0.96, at 04:00 and 05:00.

Curiously, both participants indicated that they had followed the motion of WABI reflecting their partner's physiology. However, the physiological data reveals that only P1 actually adapted her breathing behaviour beginning at trial time 03:45 and sustaining this entrainment with her partner until the end.

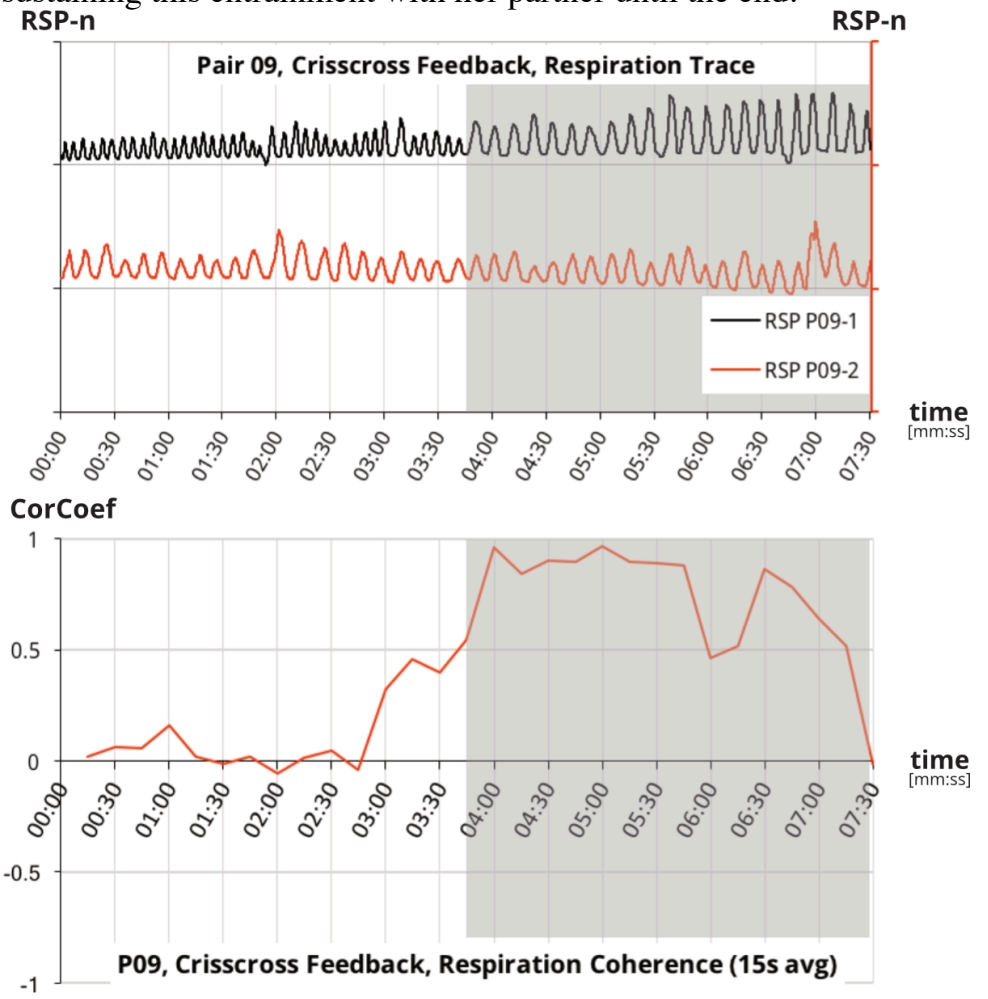

Figure 7: Respiration trace (upper graph, showing raw, normalized sensor data) and between-inhabitant Respiration Coherence (lower graph, showing Pearson Correlation Coefficient) of pair 09 in Crisscross Feedback Interaction over trial duration. Both respiration traces (upper graph) show extensive periods of auto-coherence, with each participant breathing slowly and regularly. The upper graph shows very high correlations (in-phase synchrony; grey highlight) from 03:45 onwards. Participants almost sustained synchrony until the end of the trial, with one lapse (05:45 \& 06:00) and a decline towards the end. Correlations (lower graph) above 0.5 are considered statistically significant.

In summary, participants described Crisscross Feedback Interaction as an intriguing experience that easily allowed them to match their own behaviour to that of their partner. They also explained that in this mode, regaining alignment with their partner's behaviour was easy due to the easily accessible, constant feedback of their partner's behaviour. Thus, if the architectural response is crisscrossed between two collocated people, they may become more aware of the presence and the behaviour 
of their partner. This heightened sense of the partner's presence alongside the heightened awareness of her or his (physiological) behaviour facilitates a choice of either interacting with the partner's behaviour or ignoring it. Interacting with it could for example mean to align one's behaviour with that of the partner as seen in pair 09. Moreover, this kind of feedback seems to enable the re-establishing of synchronicity between partners.

\subsubsection{Aggregate Feedback Interaction}

Three participants (3/12) preferred this trial over the other two. P09-1 explained that she did not have 'to work very hard' to achieve motion of the environment during Aggregate Feedback Interaction. She pursued her own respiration rhythm, as she indicated to not have paid 'much attention' to the environment. On the other hand, P04-1 found it more collaborative and more challenging but gave no further explanation. The most detailed description of the experience of this interaction mode was provided by P03-2 who 'felt more connected with the environment and [his] partner. Specifically, he felt 'more immersed because of the equilibrium' of the two sections of WABI. The same participant later explained that the 'accumulative process was even more interesting' than the other interaction modes.

Regarding the interaction between partners, five participants (P03-1, P03-2, P04-1, P05-2, P07-1) said that they had intentionally mimicked their partner's respiratory behaviour in this trial. Three participants interpreted the trial as more collaborative (P03-2, P04-1 \& P08-2) compared to the previous trials. However, two participants made a special point to say that they thought it 'not worth' (P03-2) or saw 'no point' (P09-2) in trying to put much effort in to this trial and synchronizing with their partner because they only had 50 per cent input into the motion of WABI.

More specifically, pair 08 shared a complex opinion of this trial between them. Both thought that this trial implied synchronisation of their behaviours. In fact, P082 explained that the synchronization with each other ' $w$ as an indicator of success in some way.' But both perceived '[...] a strange disconnect' (P08-1) between themselves and the environment. P08-1 seemed to have experienced a cognitive overload that manifested itself 'because we were concentrating on our breathing AND each other's breathing AND the tent [WABI]. Maybe it was too much.'

Pair 07 felt similarly detached, with P07-2 describing the experience of this trial in direct comparison to Crisscross Feedback Interaction as 'very disconnected.' He perceived to be disconnected from his partner in Trial 3 as it was expressed through the environment 'because it was hard to gauge where the other person was visually [referring to P1's respiratory status of breathing in or out] without actually looking at them.' For him (P2) this made it 'very odd to try and sync up with them and I found my breathing kind of suffering in that aspect, where I would forget where I was supposed to be [within the breathing cycle: breathing in or out].'

P07-1 commented on the increased effort demanded by the trial, stating that she 'felt like I was 'working' [...] to make it work properly.' P03-2 corroborated this sentiment. He described the strain introduced by Aggregate Feedback Interaction as 
causing fatigue that let to retreating into his own behaviour, leaving any attempt of behavioural alignment between both occupants to his partner:

'In the beginning, I was trying to breathe with [my partner]. But after a while I got tired and just did what I wanted to do. I thought she will adjust to my breathing. That way we will find an average.'

The challenging setup of Aggregate Feedback Interaction was partially shown in the behaviour of pair 04, although they described the opposite, speaking of increased collaboration (P04-1). Not only did their physiology never truly align (Figure 8), their behaviour indicated that they soon detached themselves from the trial and pursued their own activities, such as different seating positions (P04-1) and even extensive mobile phone use (P04-2), both shown in Figure 9. Arguably, the concurrent detachment also indicates a kind of synchrony, albeit not related to physiological behaviour.

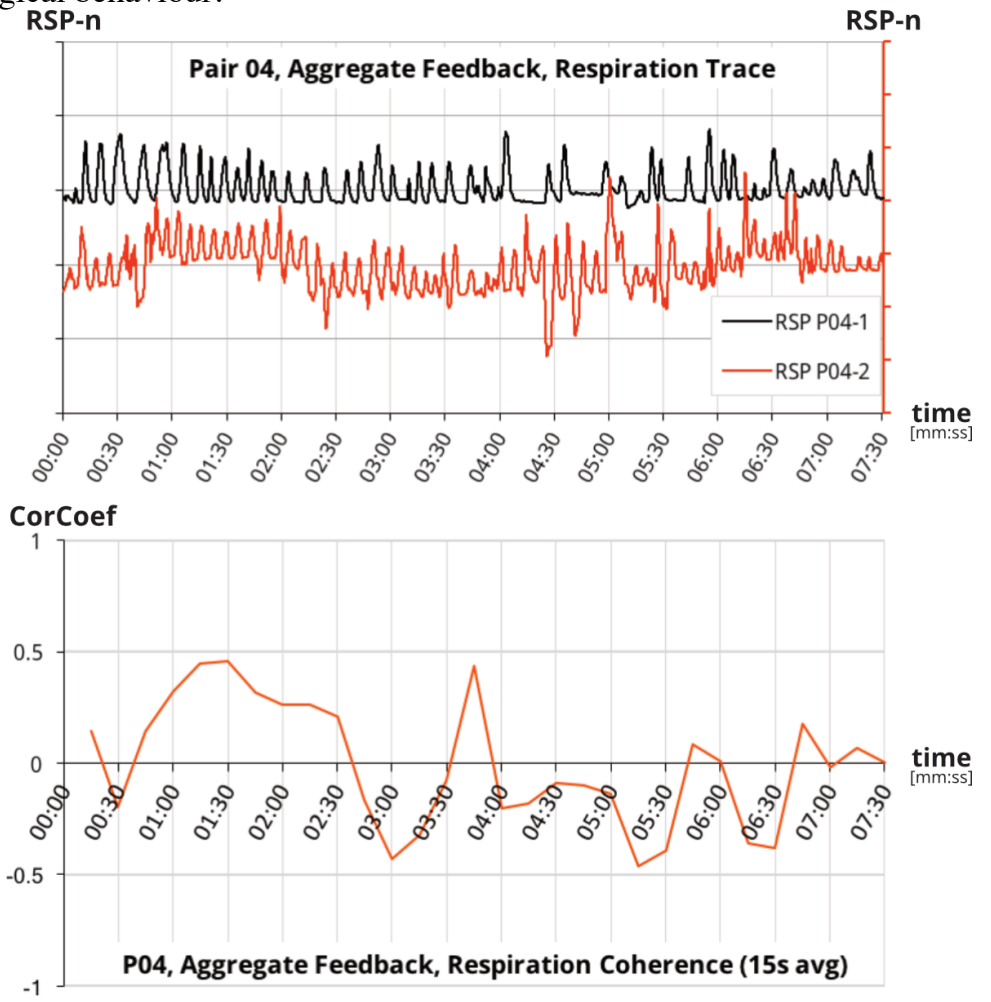

Figure 8: Respiration trace (upper graph, showing raw, normalized sensor data) and between-inhabitant Respiration Coherence (lower graph, showing Pearson Correlation Coefficient) of pair 04 in Aggregate Feedback Interaction. Both participants breathed relatively incoherently throughout the trial (top graphs), neither of them achieving extended periods of auto-coherent breathing. They were also not correlated in their respiratory behaviours (bottom graph). 


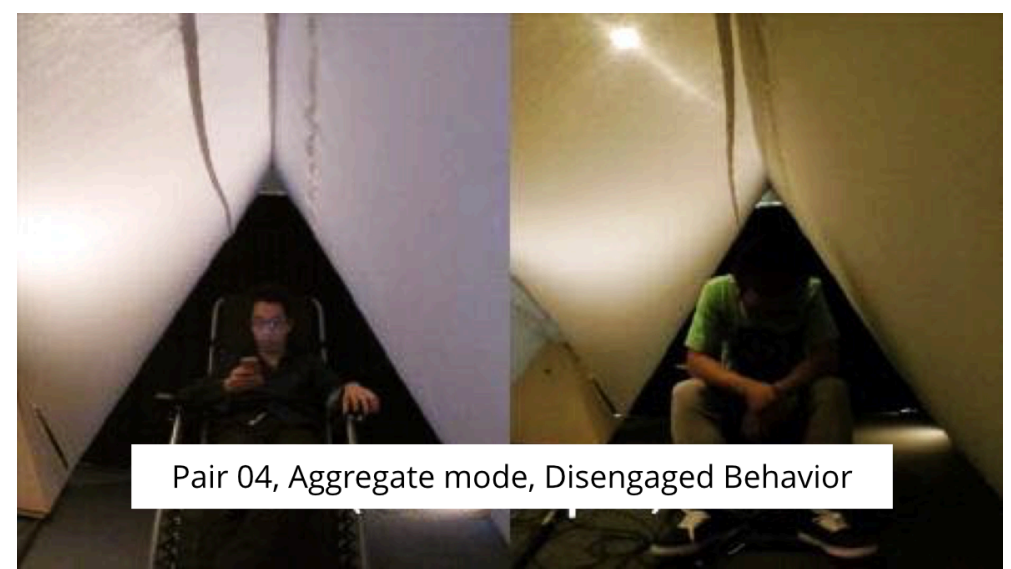

Figure 9: Pair 04 disengaged from their interaction with the environment and each other. P04-1 engaged with his mobile phone, while P04-2 changed his seating position and was in thought.

Aggregate Feedback Interaction was characterized (by two participants) as a collaborative experience. Several participants tried to mimic their partners while they seemed to be also more aware of the environment. Interactions between partners were reported to be more intentional than in the previous trials, although two participants felt that they did not want to invest too much in the environmental interaction with their partner due to their reduced degree of control over the environment, with every participant only having 50 per cent input.

This interaction mode provoked the largest variation in participant responses. Only three participants preferred this trial to the others. Many participants perceived to struggle with maintaining regular and steady breathing rhythms. Some participants felt disconnected to both environment and partner, while very few found the trial collaborative. No participant described this trial as fun, and only one participant engaged in playful behaviour.

Participant feedback indicated that this interaction mode made it difficult for them to generate smooth, consistent responses from WABI. They described it as being difficult and eventually frustrating to regain synchronicity between participants once this behavioural alignment had been lost. This feedback illustrates that when the behaviours are aggregated, the personal connection to the architectural response and the partner can be lost. A reason might be that the legibility of the individual input has disappeared. This was also revealed by some participants explaining that, due to the lack of individual feedback, they had decided during the session (after initial interaction/synchrony with the partner) to only focus on their own behaviour and disregard any environmental and partner behaviour.

To summarize, the individual accounts of Aggregate Feedback Interaction varied significantly. Some participants perceived the trial as more collaborative between 
partners or with a built-in goal to synchronize, while others felt a disconnect between themselves and their partners and the environment. Participants also found it generally more difficult to synchronize with their partners.

\section{Discussion}

Behavioural synchrony, as discussed above, has numerous benefits. As the results of our study show, architecture augmented with technology to become responsive to inhabitant behaviour can now support and, perhaps, even induce synchronous behaviour of its inhabitants. Reflecting on the results of the exploratory study, we discuss the relationship between behaviour and data mapping and how this may affect the contextual facilitation of synchrony via adaptive architecture. Doing so, we speculate how facilitating synchrony might contribute to wellbeing, therapy, and sports.

\subsection{Behaviour \& Data Mapping}

The study revealed distinct behaviours of participants in response to each of the interaction modes, which differed in their mappings of personal, physiological data to the adaptive architecture prototype WABI. The modes affected the occupants' sense of their partner and their ability to synchronise. Data mappings and technospatial affordance of synchrony also affected participants' perception of their ability and inclination to synchronise their behaviours.

Own Feedback Interaction appeared to have generally limited the extent of interactions to the participants' own section of the environment, to which their physiological data was mapped. Thus, participants tended to focus on themselves rather than on the partner despite them having a visual connection with them. Being immersed in their own feedback was individually relaxing but also seemed to decrease their sense of a physically co-present partner.

Crisscross Feedback Interaction mapped personal data to the section of the respective partner, while also allowing participants to interact with the environmental section surrounding them, which enacted the behaviour of their partner. The immersion in their partners' behaviour enabled both partners to easily coordinate their behaviours at will. They could quickly establish behavioural synchrony, sustain it, and regain it when it had been lost by mapping their own behaviour to that of their partner. Crisscross Feedback Interactions seemed to have heightened the participants' sense of their partner. Its crisscrossed data mapping encouraged or prompted them to interact with each other.

Finally, Aggregate Feedback Interaction seemed to have eliminated any perceived influence of the body on environment and partner, thereby reducing the opportunity to synchronise behaviours for extended periods. While inhabitants could still control their own body, they were unable to directly control environmental responses due to the processing (aggregation) of data. Similarly, their partner's behaviour was not legible in the environment. Thus, inhabitants could not map their 
behaviour to that of their partner. Subsequently, it was difficult to establish synchrony between partners. Or, if they initially had synchrony, they lost it quickly and could not re-establish it. Thus, some participants disengaged completely from any form of interaction with their partner.

In summary and contrary to our expectation, Aggregate Feedback was not the most synchrony inducing interaction mode, instead it made synchrony more difficult to achieve due to the limited legibility of behaviours. Participants preferred Crisscross Feedback and described it as most synchrony-facilitating and fun as a result of being increasingly aware of each other. Own Feedback mode was experienced as relaxing and self-contained because of the data remaining located within their immediate surroundings.

The data-behaviour-feedback diagram (Fig. 10) illustrates how inhabitant data maps to the environment for each interaction mode. In Own Feedback mode, a horizontal division occurs as a result of data being mapped to environmental sections surrounding each inhabitant. Inhabitants do not coordinate behaviour with each other. In Crisscross Feedback mode, data maps from one inhabitant to the environment of the other (colours cross sides). Inhabitants respond to the environment surrounding them (vertical grey underlay), which enables them to coordinate their behaviour with the other inhabitant (horizontal grey underlay). Each inhabitant judges individually whether synchrony has been established. Subsequently, a figure-eight feedback loop emerges. In Aggregate Feedback mode, a vertical division appears: data from inhabitants is equally mapped to all environmental sections (split colours). The environment coordinates its sections in a single response (upper horizontal underlay). Inhabitants (should) coordinate their behaviour (lower horizontal underlay) to generate a meaningful environmental response. The feedback between from the environment only reflects whether or not inhabitants synchronise their behaviour. It does not indicate how each inhabitant behaves, leaving them to guess how to adjust their own behaviour to align with the partner's.

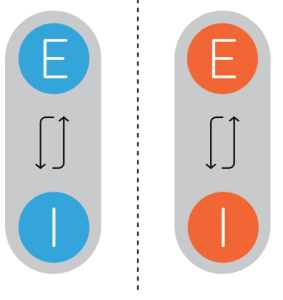

(A)
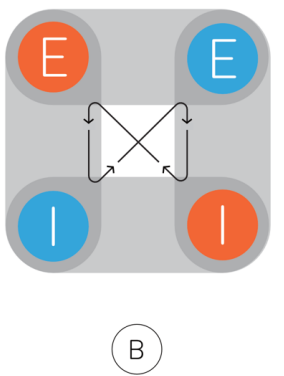
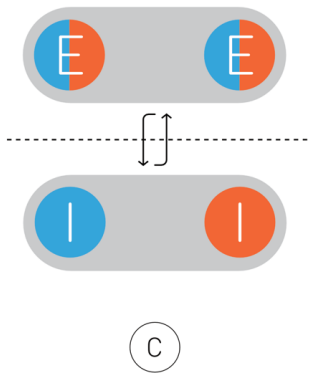

Figure 10: Data-Behaviour-Feedback Diagram. Coloured bubbles map the data of inhabitants (I) to sections of the environment $(\mathrm{E})$. The grey underlays reflect the coordination of behaviour. Black arrows indicate feedback cycle. 
The six pairs that were analysed exhibited a wide variety of behaviours both individually and in their interaction. While some were intensely focused on the breathing instructions (P07) and the interaction with their partner and the environment, others talked throughout the trials (P08) or engaged with their mobile phone (P04).

The range of behaviours indicates that environments like WABI that induce behavioural synchrony might best be designed for specific purposes and building contexts in which quiet focus on the synchronisation task can be ensured. These contexts may include as therapy, relaxation, and sport to facilitate synchrony between team mates, co-workers, romantic couples, or family members.

\subsection{From Interaction Modes to Applications}

As discussed above, interpersonal synchrony has a variety of benefits in numerous contexts. Here, we will discuss the potential applicability of the individual interaction modes to the exemplar contexts of relaxation practice, sports, and therapy.

\subsubsection{Relaxation - Yoga}

Our own work has already shown that in the practice of yoga, practitioners were keen to align their behaviour with that of the surrounding environment (Moran et al. 2016). When the environment ran in an automated movement pattern, two yoga participants attributed the status of a "master" to it. They explained that they saw the environment as providing them with guidance regarding the rate and pattern of their breathing. A projected graphic provided feedback on behavioural synchrony by fading in and out depending on the degree of synchrony. This graphical feedback of synchrony was very much appreciated by the practitioners who liked being synchronized.

In WABI, the role of the master to which inhabitants adjusted their behaviour was not an automated movement pattern, but the behaviour of the interaction partner. The equal distribution of feedback to both inhabitants was perceived as enjoyable and fun, allowing easy coordination of behaviours. Thus, Crisscross Feedback mode appears to lend itself to instructor-led sessions in which a teacher and a student synchronise their behaviour. This would allow the student to match the ideal or optimal behaviour of the teacher, while also giving the teacher feedback of the ability of the student. Similar observations of the objectification of feedback in the context of yoga were made in our earlier work (Moran et al. 2016).

\subsubsection{Sports}

Any team sport benefits from strong bonds between team members. As discussed above, synchronising behaviour can have the effect of improving one's attitude to another synchronised person. As for example described in The Sport Psychologist's Handbook: A Guide for Sport-Specific Performance Enhancement (ed. Dosil, 2008), sports psychology employs techniques, so called interventions to improve resilience to stress during competition, relaxation and recovery phases of training, 
such as autogenic training. Targeting individual athletes, some of these interventions involve breathing techniques to calm the mind and body, such as preparing for a foul shot in basketball (p.75) or controlling arousal and manage anxiety while sailing and wind surfing (pp. 467). However, sport psychologists also emphasise the importance of team coordination, for example in rugby (cf. ed. Dosil, 2008, pp.183).

To exemplify the use of spatial feedback of breathing behaviour to a group of athletes, we describe the case of rowing as coordinated behaviour, including breathing is paramount for good performance.

In rowing, it is important to synchronise one's behaviour (gross motor skills and physiological behaviour) with that of the teammates to propel the boat with maximum efficiency (cf. Schaffert and Mattes, 2015). As part of the rowing practice, it might be advantageous to use an extended version of WABI (Fig. 11) running in Crisscross Feedback mode. Teammates could take positions in it as if they sat in the boat, with the same rowers in front of and behind them.

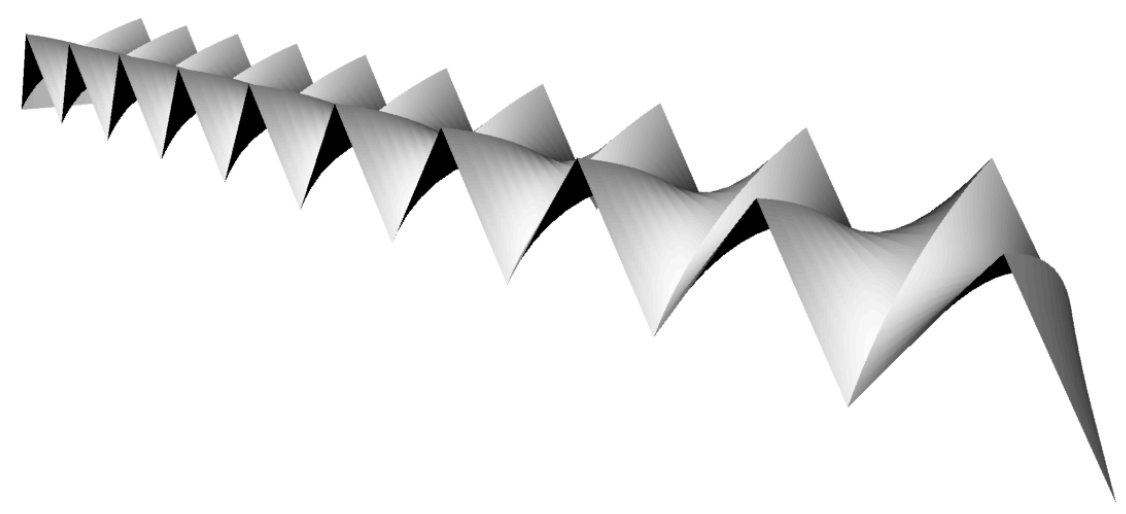

Figure 11: Arrangement of WABI sections to illustrate linear configuration of synchrony facilitating environments for multiple users.

If the data was mapped in a daisy-chain fashion (Fig. 12), each rower would respond to only one rower. The last in the chain could, for example, loop back to the first in the chain, thus closing the interactional loop. To increase the difficulty, the environment could switch to Aggregate feedback mode, which would require all teammates to be perfectly synchronised to get a legible environmental response. 


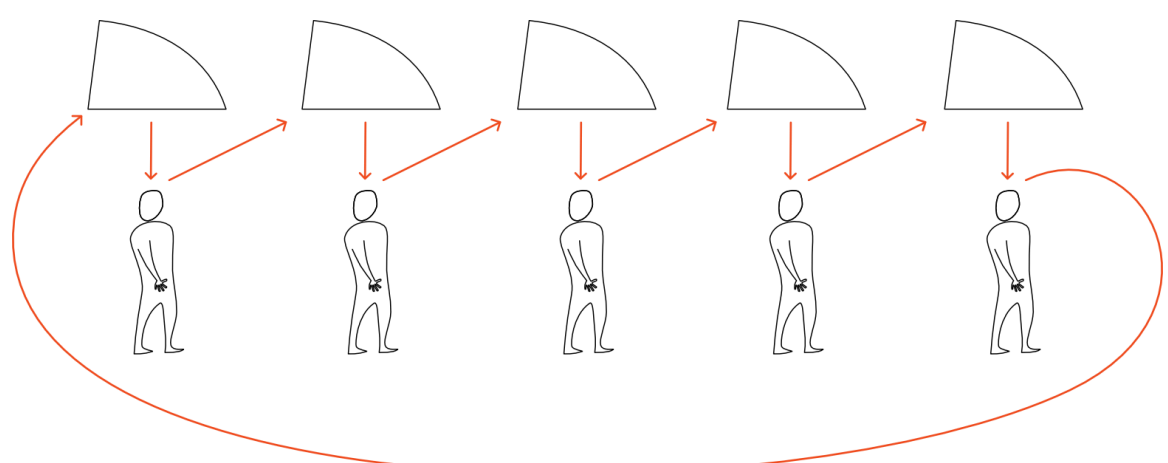

Figure 12: Daisy-chain data mapping. Data of each inhabitant is only mapped to one neighboring section (arrows). The endpoint (right) maps the last inhabitant's data back to the first section in the chain to complete the loop and link all inhabitants (or team members) together.

In such an application, the role of the environment would be similar to weight lifting or cardio equipment in a gym. Training of breathing coordination would supplement training in the boat on the water and all other strength, endurance, and technique regimes, which typically isolate muscle groups and behaviours. Breathing techniques of the individual rower and the coordination as a team would also be an isolated exercise, similar to arm curls, cardio training, or oar technique. However, if rowers where to use rowing machines in groups or as a team, a derivative of WABI could be designed and configured around such activities to provide spatial feedback about the coordination of breathing. It could, thus, augment other approaches, such as sonification of feedback, as proposed by Schaffert and Mattes (2015), who designed a feedback system called Sofirow for on-water rowing practice. Sofirow sonifies the acceleration-time-trace of the boat to the "technique training in high-performance rowing." Although rowers reported improved team cohesion and coordination, this feedback system only provides a single source of feedback based on aggregate data produced by all team members: acceleration of the boat. Unlike WABI, Sofirow does not produce individual feedback for every rower, which might further enhance coordination between team members.

\subsubsection{Therapy}

In therapeutic contexts, it might be beneficial to either synchronise therapist with patient/client or to establish synchrony between clients. The former case might help when, for example, specific behavioural patterns need to be (re)learned. In Crisscross mode, the therapist executes the ideal behaviour, while the patient/client tries to map their behaviour to the environment. Simultaneously, the therapist sees patient/client behaviour in their own section of the environment.

For situations in which synchrony between clients needs to be established, using Crisscross Feedback would also be beneficial. The environment can make its inhabitants increasingly aware of co-present other, thus making it easier to adjust to their behaviour. In addition to the physio-spatial awareness provided by the environment, 
a group of clients, once synchronised, is likely to increase their other-awareness (Asendorpf, Warkentin, and Baudonnière 1996) and improve their social perception (Miles, Nind, and Macrae 2009). We can envision this form of group therapy to apply, for example, in the work context or within families. Both of these are contexts which benefit from understanding and empathising with others. Spatially, a circular arrangement (Fig. 13) might be the most supportive configuration of group therapy or team cohesion exercises. Again, the data mapping would be in form of a daisy chain, resulting in the experience of being able to coordinate behaviour with the left and right neighbour. Ideally, this would result in the entire group synchronising their behaviour. If implemented as shown in Fig. 12, the spatial requirements for an organisation would be significant, limiting a potential deployment in the work context. With a mobile solution that folds in on itself and could be stored easily, such spatial limitations could be overcome. Further, we are considering how similar sensing and actuation technologies could be integrated into the building infrastructure directly as wall or ceiling components to facilitate regular usage.

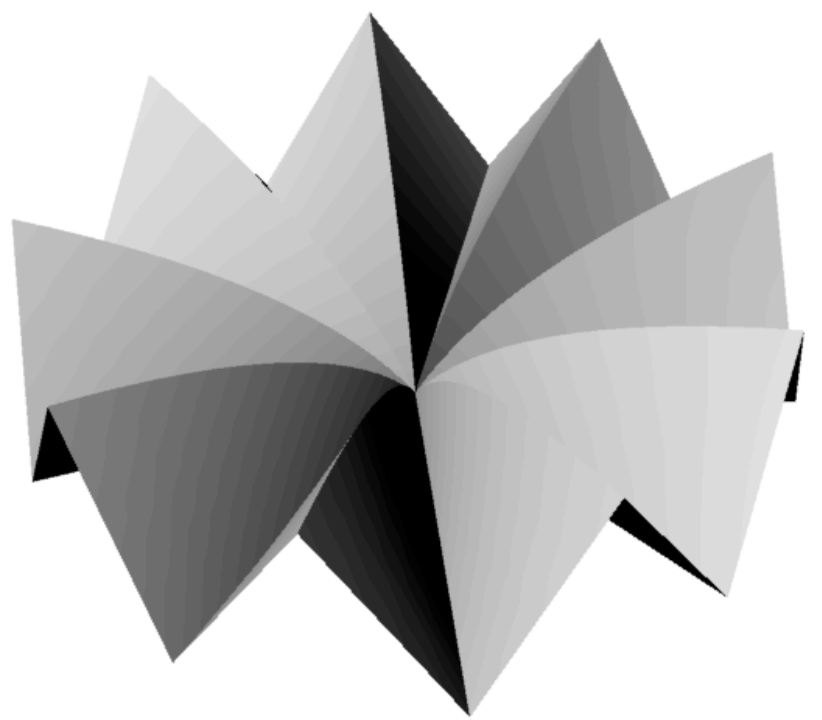

Figure 13: Arrangement of WABI sections to illustrate circular configuration of synchrony facilitating environments, for example used for group therapy or team cohesion exercises.

\subsubsection{General use}

Beyond the specific uses of relaxation, sports, and therapy, synchrony facilitating environments could also find application in public spaces, such as airports or spas. Both these venues tend to have areas in which massage chairs are lined up to provide relaxation. Similar to massage chairs synchrony facilitating environments could be used to allow groups of people, such as a family, to engage in shared breathing 
exercises. Through such exercises, they could synchronise with each other, resulting, for example, in increased pro-social behaviour and other benefits of synchrony, as discussed above, which might make, for example, a long flight less stressful.

Depending on the spatial configuration and number of WABI section used, occupants could form multiple small groups as shown in Figure 14. In this case, the adaptive system would need to identify such groups and ensure that the data mapping forms the loop between the endpoints of the daisy chain, as explained above. Limitations of such setups are the amount of space needed. However, with a critical re-design, it would be possible to optimize the use of space and materials. It would even be possible to design an environment that deploys on demand (similar to a parasol), rather than being permanently set up.
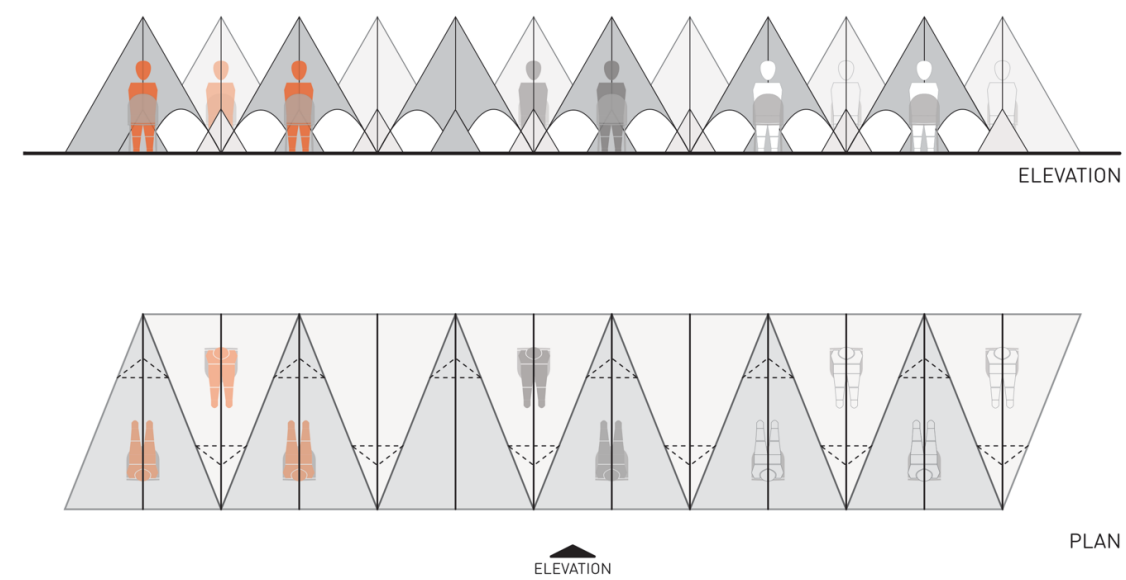

Figure 14: Plan and elevation drawings of linear arrangement of a synchrony facilitating environment, showing use by multiple small groups. Groups are defined by colours (orange, grey and white).

\subsection{Limitations}

Generalisations of our results are limited by the technological implementation of the interactions between inhabitants and environment, the number of inhabitants sharing the same space, the data being sensed, and the sample size.

Due to the bio-sensing equipment being used, inhabitants currently need to sit and are not able to move around, which would be common in other architectural spaces.

Further, only two inhabitants interacted at a time, when architectural space normally caters for varying numbers of occupants. Currently only respiration and heart rate data are being used to interact with the environment, which are highly specific data streams to sense. This data also requires a degree of skill to make the interactions valuable and enjoyable for inhabitants. 
To enable participants to acquire such skills over time, we carefully designed the procedure to increase the complexity of interaction with each trial rather than to counterbalance the conditions. However, this decision will have had an effect on participants. Though not voiced by participants, it is possible that some participants fatigued prior to the last trial (aggregate feedback), thus skewing the results in favour of the preceding trials. However, counterbalancing might have resulted in the most difficult interaction (aggregate feedback) to be first, risking that participants would not be able to interact in an enjoyable fashion and be demotivated for the remainder of the study.

Finally, the sample size of this exploratory study provides a good overview of the range of inhabitant engagement and behaviours but does not allow predictions of the most likely inhabitant behaviour to occur.

\subsection{Progressing the Work of WABI}

The work presented here generated immediate follow-on research to explore some of the emerging themes concretely. It also motivates questions of a more general, theoretical, and practical nature

\subsubsection{Immediate work}

WABI has been shown to a variety of stakeholders, including yoga teachers, care home staff and management, and a theatre director. All saw potential applications of facilitating synchrony via an adaptive space in their respective fields. We have since designed a new prototype based on the principles of WABI. This new prototype called Breathing Space also accommodates two people who now sit side-byside as opposed to facing each other. This arrangement was chosen both to reduce the footprint of Breathing Space and to make access easier. It also enables the use of by a single person if desired. Like WABI, Breathing Space kinetically responds to the respiration of its two inhabitants who can see each other's behaviour. Breathing Space has already been deployed in a local care home and we are currently analysing the results. Further work involves a deployment of Breathing Space in the studio of a yoga teacher and additional development and studies around mindfulness.

\subsubsection{Broader questions}

Considering all possible interactions between inhabitants (and between them and adaptive space) creates numerous challenges for future adaptive architecture designs, including data sources, forms of interaction, application areas, and scale. 
As technology progresses, an increasing number of sensors are embedded in the built environment as well as worn by occupants of spaces. We speculate that behavioural data from fitness trackers, mobile phones, and embedded sensors (e.g., presence, motion, emotion etc) will become generally available to computing devices. The challenge will be to map data sources to architectural behaviour/responses in such a way that the interaction is meaningful. This consists of the interaction being easily legible for the occupants, allowing them to link their behaviour to an architectural response. It also includes having a benefit to the occupants, such as synchronising their behaviours, which may lead to increased relaxation, productivity, pro-social behaviour etc.

\section{Forms of interaction}

In the study described here, the architectural space provided an interactional framework whose objectives were to facilitate synchrony between occupants of the space. Based on previous results (Jäger et al., 2017), architecture can also become a proactive agent that directly guides inhabitants to specific behaviours, such as a reduced respiratory rate. Exploring other forms of interaction, such as architecture proactively defining spaces for specific kinds of work (meeting, group work, intense focus, etc) comprises further challenges and opportunities for future work.

\section{Application: building typologies and activities}

As discussed above, we see application areas in the contexts of relaxation, sports, and therapy. Beyond these specialised areas, one challenge would be to integrate adaptive and especially synchrony-facilitating spaces into other building typologies, including homes, offices, hospitals, schools, airports etc. This would enable investigations of the applicability of such spaces "in daily use", across a broad range of activities (stationary and transient) and across different occupant demographics (age, fitness level, socio-economic status, tech-savviness, etc).

Interacting with an adaptive architectural space as described here constitutes a departure from the common experience of architectural space, which tends to be static. Mostly, people perceive architectural space passively, as a background to their activities. However, research in Human-Computer Interaction has shown that peripheral motion on a desktop screen can influence (decrease) a person's respiration rate (cf. Moraveji, 2011). Based on this research, we would anticipate even stronger effects on respiration if such peripheral motion would be spatially immersive rather than two-dimensional on a computer monitor.

\section{Scale}

The above challenges raise the issue of scalability of such interactions between occupants and architectural space on the scale of a room, a building, and the city. 
Pervading to all three scales is the technological issue of device and data format interoperability as discussed by Wilson, Hargreaves, and Hauxweell-Baldwin (2015) and, more generally related to the Internet of Things, by Milojicic, Nikoloich, and Leiba (2015). Other aspects of the scalability of WABI and its interactions are the robustness of the technology (software and hardware), sensor availability (wearable or embedded), opt-in features, and storability.

To become a deployable technology, WABI would need to become more robust in terms of hardware, which we have largely addressed with the aforementioned Breathing Space. We changed from small servo motors to linear actuators, which provide more strength and reliability. Additionally, joints and structure were made in mild steel and aluminium, adding further strength. Currently the best solution to sense respiration is a wireless belt we designed that enables occupants to move within the space. Our own tests reveal that remote sensing of breathing-offered by Novelda and its XeThru Respiration Sensor (2017) - is not yet reliable enough to provide gapless, continuous data.

Room. Taking WABI and its interactions as example, at the room level the question of occupant numbers emerges. Research is required to establish a "maximum meaningfull" number of occupants to avoid the problem of occupants not being able to identify the part of the space that responds to themselves. Similarly, it is possible that the number of occupants in a given space exceeds the number of actuators, which would potentially prevent individual feedback to each occupant. Also, when occupants change their location, the space should be able to track their movements and respond/provide feedback in spatial proximity to the occupant.

Building. Scaling up such interactions to a building introduces the challenge of maintenance of such adaptive systems as well as the portability of data. Using WABI as example once more, each section has multiple moving parts, which will require periodic maintenance. There is also a software platform that enables the interaction, which will need updates. Assuming that in a multi-storey building each floor has a setup of five to ten WABI sections, maintenance requirements would be significant for the entire building. Interactionally, the question emerges if any of the data resulting from such interactions, such as synchronicity values or user preferences are portable from one adaptive setup to the next.

City. Analogous to the building scale, a question to address at the city level is the portability of data between different buildings. This includes the crossing of building typologies (e.g., public and private) and staying within a typology (e.g., home to home, office to office). For example, does the interactional data produced at home interface in any way with a hospital or council house? Or does a friend's apartment accept and use data produced in one's own house? Additionally, public spaces may have their own agenda in trying to, for example, synchronise citizens to increase awareness of others (Asendorpf et al, 1996).

In summary, there are many challenges in the scaling up of the proposed interactions and architectural solutions. These include hardware, software, and interaction design as well as considerations of scales ranging from rooms to buildings to the city. Some of these issues have been discussed as part of a series of workshops 
titled "People, Personal Data, and Built Environment", including at the conference “Designing Interactive Systems 2017” (Schnädelbach et. al., 2017).

\section{Conclusion}

We introduced an exploratory study of a novel prototype of adaptive architecture, WABI, that facilitates behavioural synchrony between its inhabitants using highly personal data: physiological behaviour. WABI offers a variety of data mappings that affect how inhabitants interact with each other. We discussed these new spatial and interactional relationships emerging from such architectural responsiveness to multiple individuals and the data they produce. Our results show the potential for new interactional opportunities, such as using an adaptive environment to guide inhabitants to align their behaviour with that of other people sharing the same space.

Our research contributes to the knowledge of the effects of adaptive architecture on its inhabitants, which applies both to practical solutions as well as theoretical concepts of how the body relates to adaptive environments. Answering the above questions will further progress this field of research.

\section{Acknowledgments}

We thank all the participants of the study. This work was supported by the EPSRC under Grant EP/P505658 and Grant EP/M000877/1; the University of Nottingham via the Nottingham Research Fellowship "The Built Environment as the Interface to Personal Data” and Loughborough University.

\section{References}

Asendorpf, Jens B, Veronique Warkentin, and Pierre-Marie Baudonnière. 1996. "Self-Awareness and Other-Awareness. II: Mirror Self-Recognition, Social Contingency Awareness, and Synchronic Imitation..” Developmental Psychology 32 (2). American Psychological Association: 313-21. doi:10.1037/0012-1649.32.2.313.

Bell, P A, T C Greene, J Fisher, and A Baum. 1996. “Architecture, Design, and Engineering for Human Behavior.” In Environmental Psychology, 4 ed., 410-43. Fort Worth, TX: Harcourt Brace.

Berntson, G G, J T Jr Bigger, D L Eckberg, P Grossman, P G Kaufmann, M Malik, H N Nagaraja, et al. 1997. "Heart Rate Variability: Origins, Methods, and Interpretive Caveats" 34 (6): $623-48$.

Bier, Henriette, and Terry Knight. 2010. “Digitally-Driven Architecture.” Footprint 6 (Spring 2010). Delft School of Design: 1-4.

Bullivant, Lucy. 2005a. "Sky Ear, Usman Haque.” Architectural Design 75 (1). John Wiley \& Sons, Ltd.: 8-11. doi:10.1002/ad.6.

Bullivant, Lucy. 2005b. "4dspace: Interactive Architecture (Architectural Design)." Architectural Design 75 (1). London: John Wiley \& Sons: 128. http://onlinelibrary.wiley.com/doi/10.1002/ad.v75:1/issuetoc.

Bullivant, Lucy. 2005c. "D-Tower, NOX.” In Doetinchem, the Netherlands, 1998-2004, 1-4. Academy Press. doi:http://onlinelibrary.wiley.com/doi/10.1002/ad.16/pdf.

Bullivant, Lucy. 2006. Responsive Environments. London: Victoria \& Albert Museum. 
Chartrand, T L, and R van Baaren. 2009. "Human Mimicry." Advances in Experimental Social Psychology. doi:10.1016/s0065-2601(08)00405-x.

Cohen, Emma E A, Robin Ejsmond-Frey, Nicola Knight, and R I M Dunbar. 2010. "Rowers' High: Behavioural Synchrony Is Correlated with Elevated Pain Thresholds." Biology Letters 6 (1): 106-8. doi: 10.1098/rsbl.2009.0670.

Cohen, J. 1992. "A Power Primer.” Psychological Bulletin 112 (1). American Psychological Association: 155-59. doi:10.1037/0033-2909.112.1.155.

Dalton, Nicholas S, Holger Schnädelbach, Mikael Wiberg, and Tasos Varoudis. 2016. Architecture and Interaction. Edited by Nicholas S Dalton, Holger Schnädelbach, Mikael Wiberg, and Tasos Varoudis. Cham: Springer. doi:10.1007/978-3-319-30028-3.

Dosil, J. 2008. The Sport Psychologist's Handbook: A Guide for Sport-Specific Performance Enhancement (pp. 1-704). Chichester, UK: John Wiley \& Sons Ltd. http://doi.org/10.1002/9780470713174

Eng, Kynan, Andreas Babler, Ulysses Bernardet, Mark Blanchard, Marcio Costa, Tobi Delbruck, Rodney J Douglas, et al. 2003. "Ada - Intelligent Space: an Artificial Creature for the SwissExpo.02.” In, 3:4154-59. Taipei: IEEE. doi:10.1109/ROBOT.2003.1242236.

Feldman, Ruth. 2007. "Parent-Infant Synchrony." Current Directions in Psychological Science 16 (6): 340-45. doi:10.1111/j.1467-8721.2007.00532.x.

Feldman, Ruth, and Arthur I Eidelman. 2004. "Parent-Infant Synchrony and the Social-Emotional Development of Triplets" 40 (6): 1133-47. doi:10.1037/0012-1649.40.6.1133.

Feldman, Ruth, Charles W Greenbaum, and Nurit Yirmiya. 1999. "Mother-Infant Affect Synchrony as an Antecedent of the Emergence of Self-Control" 35 (1): 223-31. doi:10.1037/0012-1649.35.1.223.

Fouriezos, George, Gary Capstick, François Monette, Christine Bellemare, Matthew Parkinson, and Angela Dumoulin. 2007. "Judgments of Synchrony Between Auditory and Moving or Still Visual Stimuli" 61 (4): 277-92. doi:10.1037/cjep2007028.

Fox, Michael, and Miles Kemp. 2009. Interactive Architecture. New York: Princeton Architectural Press.

Glynn, Ruairi. 2005. "Reciprocal Space.” Interactivearchitecture.org. http://www.interactivearchitecture.org/reciprocal-space-ruairi-glynn.html.

Green, Keith Evan. 2016. Architectural Robotics. Cambridge, MA: MIT Press.

Guerra, Laura Colmenares, Todor Todoroff, and Yacine Sebti.n.d. Lungs [the Breather]. Nadine. Vol. 7. Brussels, Belgium. http://thebreather.org/index.php?page=about_en.

Hirsch, J A, and B Bishop. 1981. "Respiratory Sinus Arrhythmia in Humans: How Breathing Pattern Modulates Heart Rate..” The American Journal of Physiology 241 (4): H620-29.

Jacobs, Margot, and Jessica Findley. 2015. "Breathe." Sonicribbon.com. Accessed February 9. http://www.sonicribbon.com/sonicribbon/breathe/.

Jäger, Nils, Holger Schnädelbach, Jonathan Hale, David Kirk, and Kevin Glover. 2017. "Reciprocal Control in Adaptive Environments." Edited by Simone Barbosa. Interacting with Computers 29 (4): 512-29. doi:10.1093/iwc/iww037.

Khan, Omar. 2010. Open Columns: a Carbon Dioxide (CO2) Responsive Architecture. The 28th of the International Conference Extended Abstracts. New York, New York, USA: ACM. doi:10.1145/1753846.1754232.

Kimura, Masanori, and Ikuo Daibo. 2006. "Interactional Synchrony in Conversations About Emotional Episodes: a Measurement by 'the Between-Participants Pseudosynchrony Experimental Paradigm'.” Journal of Nonverbal Behavior 30 (3). Springer US: 115-26. doi:10.1007/s10919-006-0011-5.

Kolarevic, Branko, and Vera Parlac. 2015. Building Dynamics. London: Routledge.

Koren, L. 2008. Wabi-Sabi for Artists, Designers, Poets \& Philosophers. Imperfect Publishing. Marmot, Alexi. 2005. "Architectural Determinism. Does Design Change Behaviour?." The British Journal of General Practice 52 (476): 1-16. http://www.ncbi.nlm.nih.gov/pmc/articles/PMC1314260/pdf/12030684.pdf.

Miles, Lynden K, Louise K Nind, and C Neil Macrae. 2009. "The Rhythm of Rapport: Interpersonal Synchrony and Social Perception.” Journal of Experimental Social Psychology 45 
(3): 585-89. doi:10.1016/j.jesp.2009.02.002.

Miles, Lynden K, Louise K Nind, Zoe Henderson, and C Neil Macrae. 2010. "Moving Memories: Behavioral Synchrony and Memory for Self and Others." Journal of Experimental Social Psychology 46 (2): 457-60. doi:10.1016/j.jesp.2009.12.006.

Milojicic, D., Nikolich, P., \& Leiba, B. 2015. Standards for Tomorrow: The Internet of Things (Ubiquity symposium). Ubiquity, 2015 (November), 1-12. http://doi.org/10.1145/2822533

MindMedia. 2015. Mindmedia.com. MindMedia. http://www.mindmedia.nl/CMS/index.php.

Moran, Stuart, Nils Jäger, Holger Schnädelbach, and Kevin Glover. 2016. "ExoPranayama: a Biofeedback-Driven Actuated Environment for Supporting Yoga Breathing Practices." Personal and Ubiquitous Computing, April. Springer London, 1-15. doi:10.1007/s00779-0160910-3.

Moraveji, N., Olson, B., Nguyen, T., Saadat, M., Khalighi, Y., Pea, R., \& Heer, J. 2011. "Peripheral paced respiration: influencing user physiology during information work." 24th annual ACM symposium (pp. 423-428). New York, New York, USA: ACM. http://doi.org/10.1145/2047196.2047250

Muench, F. 2008. "The Portable StressEraser Heart Rate Variability Biofeedback Device: Background and Research." Biofeedback, 36 (1), 35-39.

Najjar, Karim, and Rames Najjar. 2016. "Bug.” Najjar-Najjar.com. Beirut. Accessed April 1. http://najjar-najjar.com/projects/?cat=5\&l=0.

Ramseyer, Fabian, and Wolfgang Tschacher. 2011. "Nonverbal Synchrony in Psychotherapy: Coordinated Body Movement Reflects Relationship Quality and Outcome..” Journal of Consulting and Clinical Psychology 79 (3). American Psychological Association: 284124. doi:10.1037/a0023419.

Sanchez-Burks, Jeffrey, Caroline A Bartel, and Sally Blount. 2009. "Performance in Intercultural Interactions at Work: Cross-Cultural Differences in Response to Behavioral Mirroring." 94 (1): 216-23. doi: $10.1037 / \mathrm{a} 0012829$.

Schaffert, N., \& Mattes, K. 2015. "Interactive Sonification in Rowing: An Application of Acoustic Feedback for On-Water Training." IEEE MultiMedia, 1-1. http://doi.org/10.1109/mmul.2015.25

Schnädelbach, Holger. 2011. "Physiological Data in Adaptive Architecture." In. London. http://scholar.google.com/scholar?q=related:aNYmS76pbmEJ:scholar.google.com/\&hl=en\&num=20\&as_sdt=0,5.

Schnädelbach, Holger, Ainojie Alexander Irune, David Kirk, Kevin Glover, and Patrick Brundell. 2012. "ExoBuilding: Physiologically Driven Adaptive Architecture." ACM Transactions on Computer-Human Interaction 19 (4). ACM: 1-22. doi:10.1145/2395131.2395132.

Schnädelbach, Holger, Kevin Glover, and Ainojie Alexander Irune. 2010. "ExoBuilding: Breathing Life Into Architecture.” In, 2010:442-51. Reykjavik. doi:10.1145/1868914.1868965.

Schnädelbach, H., Jäger, N., Nabil, S., Dalton, N., Kirk, D., \& Churchill, E. 2017. "People, Personal Data and the Built Environment." Presented at the 2017 ACM Conference on Designing Interactive Systems - DIS '17. http://doi.org/10.1145/3064857.3064864

Smith, Peter, and David J Burns. 1996. "Atmospherics and Retail Environments: the Case of the 'Power Aisle'." International Journal of Retail \& Distribution Management 24 (1). MCB UP Ltd: 7-14. doi:10.1108/09590559610107076.

Spangenberg, Eric R, B Grohmann, and David E Sprott. 2005. "It's Beginning to Smell (and Sound) a Lot Like Christmas: the Interactive Effects of Ambient Scent and Music in a Retail Setting." Journal of Business Research 58 (11): 1583-89. doi:10.1016/j.jbusres.2004.09.005.

Tanizaki, Jun'ichirō. 2001. In Praise of Shadows. Random House.

Turley, L W, and Ronald E Milliman. 2000. "Atmospheric Effects on Shopping Behavior.” Journal of Business Research 49 (2): 193-211. doi:10.1016/S0148-2963(99)00010-7.

Van Leeuwen, P, D Geue, and S Lange. 2003. "Is There Evidence of Fetal-Maternal Heart Rate Synchronization?." BMC Physiology. doi:10.1186/1472-6793-3-2.

Vidyarthi, Jay, Bernhard E Riecke, and Diane Gromala. 2012. "Sonic Cradle : Designing for an Immersive Experience of Meditation by Connecting Respiration to Music.” In, 408-17. 
New York, New York, USA: ACM. doi:10.1145/2317956.2318017.

Weiser, M. 1991. "The Computer for the 21st Century"1 (1): 19-25. doi:10.1109/MPRV.2002.993141.

Wilson, C., Hargreaves, T., \& Hauxwell-Baldwin, R. 2015. Smart homes and their users: a systematic analysis and key challenges. Personal and Ubiquitous Computing, 19(2), 463-476. http://doi.org/10.1007/s00779-014-0813-0

Novelda. (2017). X4M200 Respiration Sensor - XeThru.

Yalch, Richard F, and Eric R Spangenberg. 2000. "The Effects of Music in a Retail Setting on Real and Perceived Shopping Times." Journal of Business Research 49 (2): 139-47. doi:10.1016/S0148-2963(99)00003-X.

Yasuma, F, and J Hayano. 2004. "Respiratory Sinus Arrhythmia.” Chest Journal. doi:10.1378/chest.125.2.683. 


\section{Supplemental Material}

\section{Description of WABI}

WABI is an adaptive environment for two inhabitants that is digitally-driven using sensors and actuators. It kinetically responds to the physiological behavior (heart rate and breathing) of both its inhabitants in real time via a biofeedback mechanism. The biofeedback link - a loop consisting of inhabitant behavior, environment sensing, environment response, inhabitant sensing, and adjusted inhabitant behavior (Schnädelbach 2011 explains this loop in more detail) - between each inhabitant and WABI establishes both a coupling between each inhabitant and the environment, as well as between inhabitants. This unique physio-spatial relationship between inhabitants, as modulated by the physical structure, is its primary achievement: WABI enables two inhabitants to be collocated per our definition of collocation in architecture. Two people can be present within the same spatial unit at the same time. They are within interactional distance and share an activity. Depending on the specific data processing performed by WABI, the experience for inhabitants can vary distinctly, as we describe above.

The following section contains a description of the physical, technical, and interactional features of WABI, including descriptions of the structure and motion mechanism, the hardware and software of this prototype, and finally the range of interactions this design enables.

\section{Hardware}

The floor plan (Figure S-1) consists of two triangles, which are 180 degrees rotated against each other. In this tessellated shape, the outline of WABI in its current form resembles a rhombus. From the sharp angle of each triangle, an aluminum 'spine' rises diagonally across the triangular floor plan (Figure S-1) up to height of $210 \mathrm{~cm}$ above the shortest side of the ground triangle (Figure S-2). Two steel tubes connect the spine with two steel tubes on the ground, which form the outline of WABI. Steel tubes of varying diameters form a triangular frame that makes WABI structurally independent (Figure S-3).

Wooden tetrahedral housing units encase the movement mechanism. Three fabric-covered LED lights illuminate the prototype with a soft yellow glow while inhabitants are present. Two LED lights are located behind each inhabitant on the lefthand side of WABI. The lights are clamped to the vertical steel frame. The LED light providing lighting from above, is suspended from an aluminium pole, which is attached to the ceiling structure.

At its highest point, the structure is about $210 \mathrm{~cm}$ tall and about $265 \mathrm{~cm}$ wide. Overall, it is about $365 \mathrm{~cm}$ long. On the lower, narrow end, sits a tetrahedral wooden box, which houses the servomotor and arm assembly (Figure 6) alongside a small video camera to record participants during trials. 


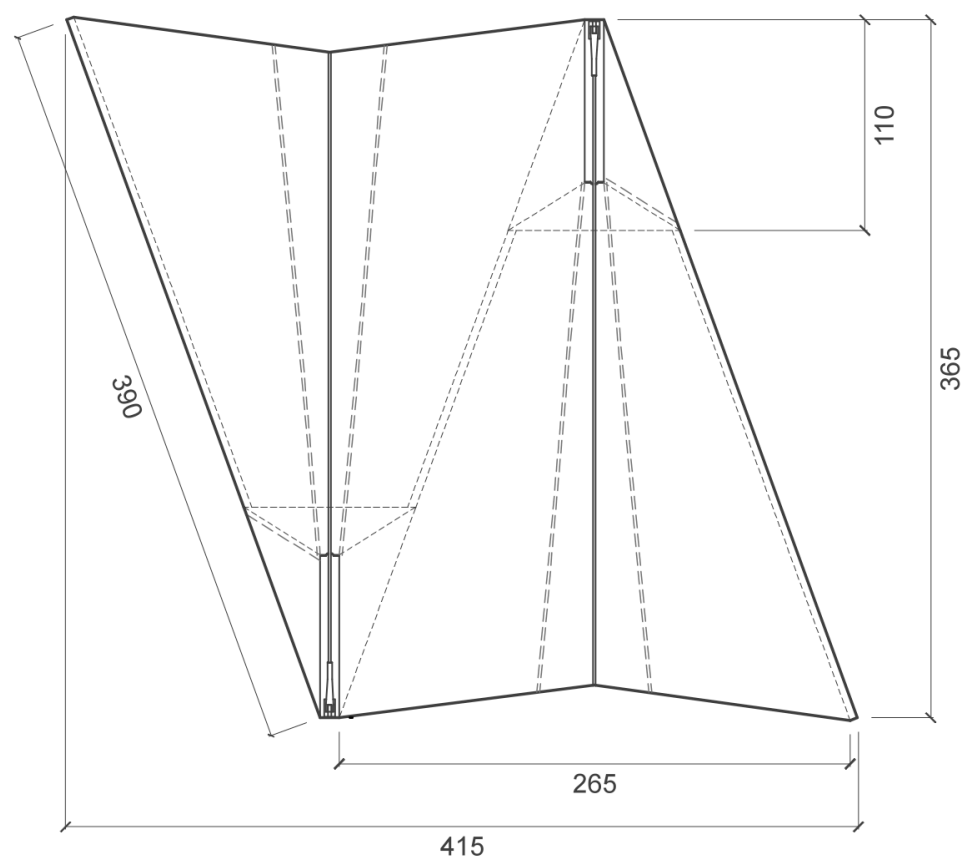

Figure S-1: Triangular, tessellated floor plan of WABI. Dimensions are approximate in centimeters.

Each inhabitant sits under one spine, over which white jersey fabric stretches. The fabric is assembled in panels, which are connected with hook and loop tape. The modularity of the fabric allows parts of the fabric to be exchanged if WABI undergoes a reconfiguration or extension. The jersey fabric constitutes the skin of the building and partially encloses the inhabitants (Figure 4). WABI is closed on two of its four sides, always to the left of each participant. The remaining two sides are open and provide entrances for each inhabitant.

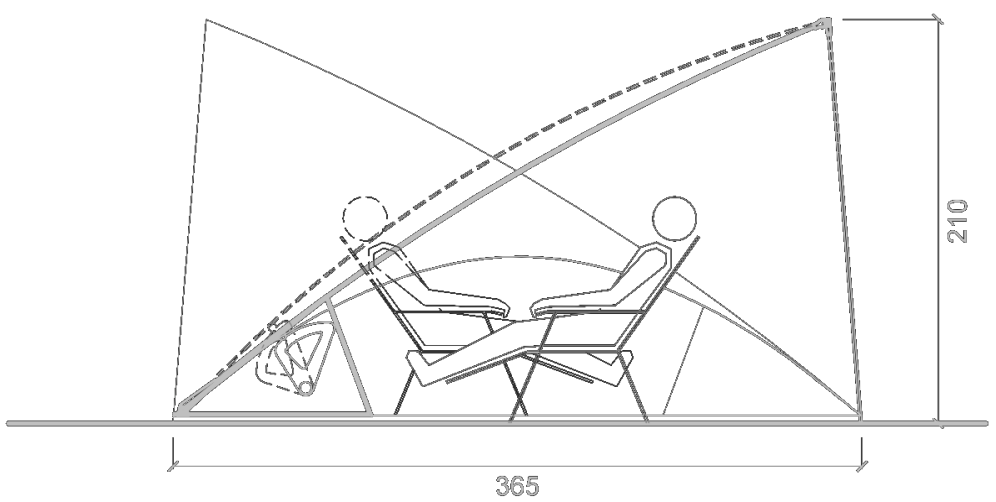


Figure S-2: Section and Side Elevation. Dimensions are approximate in centimeters.

The initial configuration accommodates two inhabitants who are facing each other (Figure S-2). The triangular floor plan (Figure S-1) consisting of a symmetrical forty-degree (40 degree) triangle, is tessellated to achieve the current spatial arrangement in which both inhabitants sit diagonally across from each other. Thus, they are facing each other, sitting in a section of the space each. The sections are separately actuated but spatially connected (Figure S-2 and S-3), allowing visual awareness of the other inhabitant.

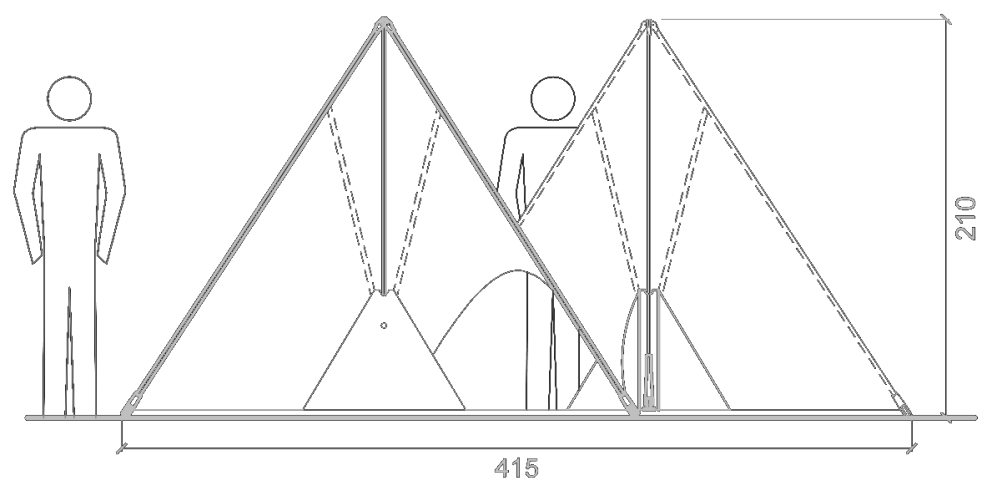

Figure S-3: Elevation (front and back) of WABI. Dimensions are approximate in centimeters.

Name

The name WABI describes the nature of the physical and interactive features of this biofeedback space. The word 'wabi' derives from the elusive Japanese term 'wabi-sabi', a concept of imperfect, impermanent, and incomplete beauty. (see Tanizaki (Tanizaki 2001) and Koren (Koren 2008) for details). Wabi alone refers to a rough, unfinished simplicity or understated elegance. But it also sometimes relates to 'quirks and anomalies arising from the process of construction' (http://en.wikipedia.org/wiki/Wabi-sabi). 